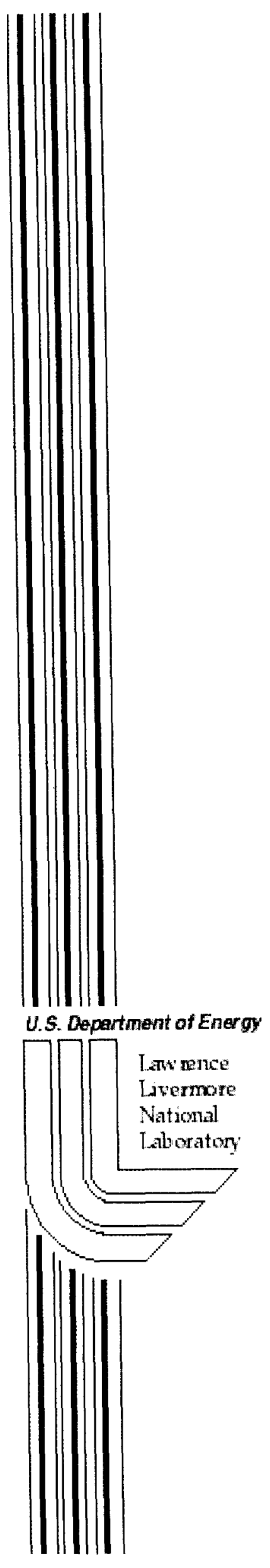

\title{
A Consistent Approach to Solving the Radiation Diffusion Equation
}

\author{
J.H. Hammer, M.D. Rosen
}

This article was submitted to $44^{\text {th }}$ Annual Meeting of the Division of Plasma Physics, Orlando, Florida, November 11-15, 2002

\section{November 6, 2002}




\section{DISCLAIMER}

This document was prepared as an account of work sponsored by an agency of the United States Government. Neither the United States Government nor the University of California nor any of their employees, makes any warranty, express or implied, or assumes any legal liability or responsibility for the accuracy, completeness, or usefulness of any information, apparatus, product, or process disclosed, or represents that its use would not infringe privately owned rights. Reference herein to any specific commercial product, process, or service by trade name, trademark, manufacturer, or otherwise, does not necessarily constitute or imply its endorsement, recommendation, or favoring by the United States Government or the University of California. The views and opinions of authors expressed herein do not necessarily state or reflect those of the United States Government or the University of California, and shall not be used for advertising or product endorsement purposes.

This is a preprint of a paper intended for publication in a journal or proceedings. Since changes may be made before publication, this preprint is made available with the understanding that it will not be cited or reproduced without the permission of the author.

This report has been reproduced directly from the best available copy.

Available electronically at http://www.doe.gov/bridge

Available for a processing fee to U.S. Department of Energy and its contractors in paper from

U.S. Department of Energy

Office of Scientific and Technical Information

P.O. Box 62

Oak Ridge, TN 37831-0062

Telephone: (865) 576-8401

Facsimile: (865) 576-5728

E-mail: reports@adonis.osti.gov

Available for the sale to the public from

U.S. Department of Commerce

National Technical Information Service

5285 Port Royal Road

Springfield, VA 22161

Telephone: (800) 553-6847

Facsimile: (703) 605-6900

E-mail: orders@ntis.fedworld gov

Online ordering: http://www.ntis.gov/ordering.htm

OR

Lawrence Livermore National Laboratory

Technical Information Department's Digital Library

http://www.llnl.gov/tid/Library.html 


\title{
A consistent approach to solving the radiation diffusion equation
}

\author{
James H. Hammer and Mordecai D. Rosen \\ Lawrence Livermore National Laboratory, Livermore, CA 94551
}

\begin{abstract}
Diffusive X-ray-driven heat waves are found in a variety of astrophysical and laboratory settings, e.g. in the heating of a hohlraum used for ICF, and hence are of intrinsic interest. However, accurate analytic diffusion wave (also called Marshak wave) solutions are difficult to obtain due to the strong non-linearity of the radiation diffusion equation. The typical approach is to solve near the heat front, and by ansatz apply the solution globally. This works fairly well due to "steepness" of the heat front, but energy is not conserved and it does not lead to a consistent way of correcting the solution or estimating accuracy. We employ the steepness of the front through a perturbation expansion in $\varepsilon=\beta /(4+\alpha)$, where the internal energy varies as $T^{\beta}$ and the opacity varies as $T^{-\alpha}$. We solve using an iterative approach, equivalent to asymptotic methods that match outer (away from the front) and inner (near the front) solutions. Typically $\varepsilon<0.3$. Calculations are through first order in $\varepsilon$ and are accurate to $\sim 10 \%$, which is comparable to the inaccuracy from assuming power laws for material properties. We solve for supersonic waves with arbitrary drive time history, including the case of a rapidly cooling surface, and generalize the method to arbitrary temperature dependence of opacity and internal energy. We also solve for subsonic waves with drive temperature varying as a
\end{abstract}


power of time. In the subsonic case, the specific heat, (pressure/density) and opacity are each assumed to vary as density to a small power, of order $\varepsilon$. We find the theory compares well with radiation hydrodynamics code calculations of the heat front position, absorbed flux and ablation pressure.

PACS 52.25.Os, 52.57.Bc, 95.30.Jx

\section{INTRODUCTION}

Radiation heat waves, or Marshak waves, play an important role in energy transport for many astrophysical and laboratory plasmas [1-4]. Of particular interest is the role of Marshak waves in the energy balance of laser, z-pinch and heavy ion beam hohlraums for inertial confinement fusion (ICF) and high energy density physics experiments. In these experiments, a power source, e.g. a laser, delivers energy to the interior of a high $\mathrm{Z}$ cavity that is converted to $\mathrm{X}$-rays [4]. Typically, most of the energy is absorbed in a thin, diffusively-heated layer on the hohlraum interior surface, and reemission from the heated layer sets the radiation temperature achieved in the hohlraum. The physics of the Marshak-wave-heated layer is therefore of critical importance for these systems. Marshak waves also appear in a variety of other laboratory experiments and astrophysical contexts, e.g. precursors to radiating shock waves.

A typical Marshak wave solution for the temperature profile within heated material is shown in Fig. (1). A long-recognized property of the Marshak wave is the "steepness" of the heat front due primarily to the strong non-linear temperature dependence of the radiation energy density $\sim T^{4}$. In this paper we develop an analytic 
theory of Marshak waves exploiting the "steepness" to construct a perturbation theory. Corresponding to the steepness is a small parameter $\varepsilon=\beta /(4+\alpha)$, where the internal energy varies as $T^{\beta}$ and the opacity varies as $T^{-\alpha}$. A consistent theory can be built up order-by-order in $\varepsilon$, with the benefits of good accuracy and order-by-order energy conservation.

Modern computational codes can readily generate numerical solutions to the nonlinear partial differential equations goveming Marshak waves, yet an analytic theory is still of considerable value for developing insight into the problem. An analytic theory also allows rapid scoping calculations for hohlraum design, guides hohlraum materialoptimization studies, and gives values for the time and angle-dependent albedo ( $x$-ray reemission coefficient) of a hohlraum wall for use in interpretation of experiments. The latter topic will be addressed in a follow-on publication. Design of experiments scaling one radiation-hydrodynamic system to another, e.g. scaled ICF ignition capsules, can also benefit from analytic models [5]. Finally, the methodology developed here can be applied to other nonlinear conduction problems, e.g. electron conduction heat fronts in laser-heated plasmas.

The basic equation for supersonic, diffusive radiative transport in one dimension is

$$
\rho \frac{\partial e}{\partial t}=\frac{4}{3} \frac{\partial}{\partial x} \frac{1}{K \rho} \frac{\partial \sigma T^{4}}{\partial x}
$$

where $e$ is the internal energy per unit mass, $\rho$ is the density, $T$ is the temperature, $\sigma$ is the Stefan-Boltzmann constant, $K$ is the Rosseland-mean opacity, $t$ is time, and $x$ is the spatial coordinate. $e$ and $K$ are specified functions of $\rho, T$. for given materials. For supersonic transport we consider the case of constant $\rho$, since, by definition, the radiation 
is progressing faster than the time scale for hydrodynamic motion to give rise to density changes. Typical boundary conditions for the solution of equation (1) are either a known surface temperature or incident radiation flux as a function of time. If the material has finite thickness, then the temperature or incident flux must be specified on both boundaries. We will concentrate on the case with a single boundary, i.e. a semi-infinite slab of material whose surface conditions are given. Solutions of equation (1) starting from a cold slab show progression of a distinct heat front through the material, i.e. the temperature rises abruptly from the ambient values at the front. The temperature as a function of position is characteristically steep near the heat front (Fig 1.) due to the strong non-linearity of the equation.

We can use the "steepness" of the temperature profile to gain insight into the nature of the solution through some simple arguments. Since the temperature is spatially flat away from the front, the left hand side of eq. (1) tends to be largest near the front where $T$ and $e$ vary rapidly. An exception to this occurs when the surface temperature changes rapidly, as we discuss in section II.C below. In the more typical case, however, we can consider the left hand side to be small away from the front, so the temperature profile approximately satisfies the steady-state heat flow equation

$$
\frac{\partial}{\partial x} \frac{1}{K \rho} \frac{\partial \sigma T^{4}}{\partial x}=0
$$

Defining the optical depth as $\tau=\int K \rho d x$, the equation can be rewritten as

$$
\frac{\partial^{2} T^{4}}{\partial \tau^{2}}=0
$$

with solutions $T=T_{S}\left(1-\tau / \tau_{F}\right)^{1 / 4}$, where $T_{S}$ is the surface temperature and $\tau_{F}$ is the optical depth to the heat front. The $1 / 4$ root in the solution shows why the temperature 
varies steeply near the front, as we assumed. For the simple case of constant opacity, we could also write $T=T_{S}\left(1-x / x_{F}\right)^{1 / 4}$.

We can find the advance of the front in time by considering conditions near the front. The radiative power per unit area reaching the heat front is $-\frac{4}{3} \frac{1}{K \rho} \frac{\partial \sigma T^{4}}{\partial x} \cong \frac{4}{3} \frac{1}{K \rho} \frac{\sigma T_{S}^{4}}{x_{F}}$ from our approximate solution for the temperature profile. The radiative power supplies the internal energy increase caused by motion of the front, i.e., as the front advances a greater mass of material is heated to the (nearly uniform) temperature behind the front. For the case of $T_{S}$, constant in time, this gives

$$
\rho e_{S} \frac{\partial x_{F}}{\partial t} \cong \frac{4}{3} \frac{1}{K \rho} \frac{\sigma T_{S}^{4}}{x_{F}}
$$

where $e_{S}$ is the internal energy at the surface temperature. We can easily solve this for the heat front position,

$$
\frac{\partial}{\partial t} \frac{x_{F}{ }^{2}}{2}=\frac{4}{3} \frac{1}{K \rho^{2}} \frac{\sigma T_{S}^{4}}{e_{S}}, \text { so } x_{F}=\sqrt{\frac{8}{3 K \rho^{2}} \frac{1}{e_{S}} t}
$$

showing the characteristic $t^{1 / 2}$ dependence of diffusion waves.

In succeeding sections, we exploit the "steepness" of Marshak waves to construct a systematic perturbation solution for a number of cases of interest. The result that the lowest order solution is the steady-state solution lies at the heart of the analysis, just as in our rough arguments above. 
The paper proceeds in Sec. II with the derivation of analytic solutions to supersonic Marshak waves. We consider the case of arbitrary time variation of the surface temperature, with material properties varying as $\rho, T$ power laws in Sec. IIa, and comparisons of the perturbation solution with the exact Henyey solution in Sec IIb. In Sec. IIc, we consider the case when the surface temperature varies rapidly enough to modify the lowest order solution. This is of particular interest for rapidly cooling surfaces, such as when the driving power source is turned off and the net flux into the material vanishes. Our treatment of supersonic waves concludes in Sec. IId with the generalization of the analysis of IIa to the case of non-power law material properties. In Sec. III, we construct perturbation solutions to the subsonic equations for the case that the surface temperature varies as $t^{k}$, where self-similar solutions can be sought. Our subsonic solutions include the hydrodynamic flow solution as well as values for the ablation pressure.

\section{SUPERSONIC WAVES}

\section{A. Power-law material properties}

In this section we analyze equation (1) under the assumption that

$$
e=f T^{\beta} \rho^{-\mu}, \quad \frac{1}{K}=g T^{\alpha} \rho^{-\lambda}
$$

with $f$, $g$ constants. Inserting eq. (6) into eq. (1), together with $\rho=$ constant gives 


$$
\begin{aligned}
& \frac{\partial}{\partial t} T^{\beta}=C \frac{\partial^{2}}{\partial x^{2}} T^{4+\alpha} \\
& C=\frac{16}{(4+\alpha)} \frac{g \sigma}{3 f \rho^{2-\mu+\lambda}}
\end{aligned}
$$

Next we introduce the dimensionless variable, $\zeta(x, t)=\frac{T^{4+\alpha}}{T_{S}^{4+\alpha}}$, and the quantity $H(t)=T_{S}^{4+\alpha}$, where $T_{S}$ is the time-dependent surface temperature. Using these definitions in eq. (7) we obtain

$$
\begin{aligned}
& \frac{\partial}{\partial t} H^{\varepsilon} \zeta^{\varepsilon}=C H \frac{\partial^{2}}{\partial x^{2}} \zeta \\
& \varepsilon=\frac{\beta}{4+\alpha}
\end{aligned}
$$

The parameter $\varepsilon$ defined in eq. (8) plays an important role in our theory. The smallness of $\varepsilon$ (typically $<0.3$ for high $\mathrm{Z}$ materials in ICF hohlraums) is directly connected to the steepness of the temperature profiles. We will employ $\varepsilon$ as our expansion parameter.

It is also useful to introduce a dimensionless spatial variable $y=\frac{x}{x_{F}}$, where $x_{F}$ is the time-dependent heat front position. Our boundary conditions for $\zeta(y, t)$ are then

$$
\begin{aligned}
& \zeta(0, t)=1 . \\
& \zeta(1, t)=0,\left.\quad \frac{\partial \zeta}{\partial y}\right|_{y=1}=0 .
\end{aligned}
$$

The last condition comes from the requirement that the heat flux as well as the temperature must vanish at the front. Using $y$ as the independent variable means we must transform the derivative according to

$$
\left.\frac{\partial}{\partial t}\right|_{x}=\left.\frac{\partial}{\partial t}\right|_{y}+\left.\frac{\partial y}{\partial t}\right|_{x} \frac{\partial}{\partial y}=\left.\frac{\partial}{\partial t}\right|_{y}-\frac{y}{x_{F}} \frac{\partial x_{F}}{\partial t} \frac{\partial}{\partial y}
$$


so that eq(8) becomes

$$
\left(\frac{\partial}{\partial t} H^{\varepsilon}\right) \zeta^{\varepsilon}+H^{\varepsilon} \frac{\partial}{\partial t} \zeta^{\varepsilon}-H^{\varepsilon} \frac{y}{x_{F}} \frac{\partial x_{F}}{\partial t} \frac{\partial}{\partial y} \zeta^{\varepsilon}=\frac{C H}{x_{F}^{2}} \frac{\partial^{2}}{\partial y^{2}} \zeta
$$

A final variable transformation is to a dimensionless time $s=\int \frac{C H^{1-\varepsilon}}{x_{F}{ }^{2}} d t$. With this change eq. (11) becomes

$$
\varepsilon \frac{1}{H} \frac{\partial H}{\partial s} \zeta^{\varepsilon}+\frac{\partial \zeta^{\varepsilon}}{\partial s}-\frac{y}{x_{F}} \frac{\partial x_{F}}{\partial s} \frac{\partial \zeta^{\varepsilon}}{\partial y}=\frac{\partial^{2} \zeta}{\partial y^{2}}
$$

We are nearly ready to apply perturbation techniques, since $\frac{\partial \zeta^{\varepsilon}}{\partial y}=\frac{\varepsilon}{\zeta^{1-\varepsilon}} \frac{\partial \zeta}{\partial y}$, etc. so that the left hand side of eq. (12) appears to be a correction of order $\varepsilon$. The difficulty comes near the front where $\frac{1}{\zeta^{1-\varepsilon}} \frac{\partial \zeta}{\partial y} \rightarrow \infty$ and the expansion technique breaks down (the $\frac{\partial \zeta^{\varepsilon}}{\partial s}$ term goes to zero at the front since $\zeta(1, s)=0$ for all $s)$. One approach we have used is to find the solution away from the front through an expansion in $\varepsilon$, as well as a solution near the front found for $(1-y)<<1$, then match in the overlap region where both expansions are valid. An algebraically simpler, but equivalent, "trick" is to add a perfect derivative term to both sides of eq. (12) that guarantees that the left hand side is uniformly of order $\varepsilon$. By inspection, we see that the term to add is $\frac{1}{x_{F}} \frac{\partial x_{F}}{\partial s} \frac{\partial \zeta^{\varepsilon}}{\partial y}$ so that eq. 12 becomes

$$
\varepsilon \frac{1}{H} \frac{\partial H}{\partial s} \zeta^{\varepsilon}+\frac{\partial \zeta^{\varepsilon}}{\partial s}+\frac{(1-y)}{x_{F}} \frac{\partial x_{F}}{\partial s} \frac{\partial \zeta^{\varepsilon}}{\partial y}=\frac{\partial}{\partial y}\left(\frac{\partial \zeta}{\partial y}+\frac{1}{x_{F}} \frac{\partial x_{F}}{\partial s} \zeta^{\varepsilon}\right)
$$

Note that $\frac{(1-y)}{x_{F}} \frac{\partial x_{F}}{\partial s} \frac{\partial \zeta^{\varepsilon}}{\partial y}=\varepsilon \frac{(1-y)}{x_{F} \zeta^{1-\varepsilon}} \frac{\partial x_{F}}{\partial s} \frac{\partial \zeta}{\partial y} \rightarrow \varepsilon \times \Theta(1)$ term even as $y \rightarrow 1$, and the other terms on the left hand side of 13 area also order $\varepsilon$ (as long as $\frac{1}{H} \frac{\partial H}{\partial s}$ is of order unity - 
deviations from this are discussed in Sec IIc). We will apply this trick and its variations throughout the paper. The lowest order equation comes from neglecting the left hand side,

$$
\frac{\partial}{\partial y}\left(\frac{\partial \zeta}{\partial y}+\frac{1}{x_{F}} \frac{\partial x_{F}}{\partial s} \zeta^{\varepsilon}\right)=0
$$

Integrating Eq. (14) and applying boundary conditions from eq. (9) gives

$$
\frac{\partial \zeta}{\partial y}+\frac{1}{x_{F}} \frac{\partial x_{F}}{\partial s} \zeta^{\varepsilon}=0
$$

Eq. (15) can be integrated, applying the boundary condition $\zeta(0, s)=1$

$$
\zeta=\left(1-(1-\varepsilon) \frac{1}{x_{F}} \frac{\partial x_{F}}{\partial s} y\right)^{\frac{1}{1-\varepsilon}}
$$

Applying the boundary condition $\zeta(1, s)=0$ gives the lowest order equation for the heat front $(1-\varepsilon) \frac{1}{x_{F}} \frac{\partial x_{F}}{\partial s}=1$, so we have the lowest order solution

$$
\zeta=(1-y)^{\frac{1}{1-\varepsilon}} \cong 1-y
$$

Note this is the steady state solution discussed in Sec I (in normalized variables). The last form of $\zeta$ in eq. (17) is sufficiently accurate to substitute into the left hand side of eq (13) to obtain the first order corrections.

$$
\varepsilon \frac{1}{H} \frac{\partial H}{\partial s} \zeta^{\varepsilon}-\frac{\varepsilon}{x_{F}} \frac{\partial x_{F}}{\partial s} \zeta^{\varepsilon}=\frac{\partial}{\partial y}\left(\frac{\partial \zeta}{\partial y}+\frac{1}{x_{F}} \frac{\partial x_{F}}{\partial s} \zeta^{\varepsilon}\right)
$$

In integrating eq(18), we can replace $\zeta^{\varepsilon}$ by 1 to leading order since

$$
\begin{gathered}
\int_{0} \zeta^{\varepsilon} d y=\int_{0}(1-y)^{\varepsilon} d y=\frac{1}{1-\varepsilon} \cong 1 \text {. Integration of eq.(18) then gives } \\
\varepsilon(y-1)\left(\frac{1}{H} \frac{\partial H}{\partial s}-\frac{1}{x_{F}} \frac{\partial x_{F}}{\partial s}\right)=\frac{\partial \zeta}{\partial y}+\frac{1}{x_{F}} \frac{\partial x_{F}}{\partial s} \zeta^{\varepsilon}
\end{gathered}
$$


where we have applied boundary conditions from eq. (9). Eq. (19) can be integrated by dividing through by $\zeta^{\varepsilon}$, using $\zeta^{-\varepsilon} \frac{\partial \zeta}{\partial y}=\frac{1}{1-\varepsilon} \frac{\partial \zeta^{1-\varepsilon}}{\partial y}$, substituting $\zeta^{\varepsilon}=1$ and $\frac{1}{x_{F}} \frac{\partial x_{F}}{\partial s}=1$ in the order $\varepsilon$ corrections, and applying the boundary condition $\zeta(0, s)=1$. The result is a solution accurate to order $\varepsilon$ :

$$
\zeta=\left(1-(1-\varepsilon) \frac{1}{x_{F}} \frac{\partial x_{F}}{\partial s} y+\varepsilon\left(1-\frac{1}{H} \frac{\partial H}{\partial s}\right)\left(y-\frac{y^{2}}{2}\right)\right)^{\frac{1}{1-\varepsilon}}
$$

We can now find the heat front position through order $\varepsilon$ by applying the boundary condition $\zeta(1, s)=0$,

$$
(1-\varepsilon) \frac{1}{x_{F}} \frac{\partial x_{F}}{\partial s}=1+\frac{\varepsilon}{2}-\frac{\varepsilon}{2} \frac{1}{H} \frac{\partial H}{\partial s}
$$

The procedure can be iterated to generate solutions of order $\varepsilon^{2}$. Omitting the straightforward details, we find

$$
(1-\varepsilon) \frac{1}{x_{F}} \frac{\partial x_{F}}{\partial s}=1+\frac{\varepsilon}{2}-\frac{\varepsilon}{2}(1-\varepsilon) \frac{1}{H} \frac{\partial H}{\partial s}+\frac{\varepsilon^{2}}{6}\left(1+\frac{1}{H} \frac{\partial H}{\partial s}+\frac{\partial}{\partial s} \frac{1}{H} \frac{\partial H}{\partial s}\right)
$$

Since $\frac{\varepsilon^{2}}{6}$ is usually quite small, we adopt

$$
\frac{1}{x_{F}} \frac{\partial x_{F}}{\partial s}+\frac{\varepsilon}{2} \frac{1}{H} \frac{\partial H}{\partial s}=\frac{(1+\varepsilon / 2)}{(1-\varepsilon)}
$$

as sufficiently accurate (again excluding rapid temperature variation). At this point, it is convenient to change back to the dimensional time variable, so eq.(23) gives

$$
\frac{x_{F}}{C H^{1-\varepsilon}} \frac{\partial x_{F}}{\partial t}+\frac{\varepsilon}{2} \frac{x_{F}^{2}}{C H^{2-\varepsilon}} \frac{\partial H}{\partial t}=\frac{(1+\varepsilon / 2)}{(1-\varepsilon)}
$$

Eq. (24) can be integrated in time to give

$$
x_{F}^{2}=\frac{(2+\varepsilon)}{(1-\varepsilon)} C H^{-\varepsilon} \int H d t
$$


where $\mathrm{C}$ is defined in eq. (7). Eq. (25) is one of our principal results: the heat front position for arbitrary time history of the surface temperature. We can also find the absorbed heat flux at the surface

$$
F=-\left.\frac{4}{3} \frac{1}{K \rho} \frac{\partial \sigma T^{4}}{\partial x}\right|_{x=0}=-\left.\frac{16}{3(4+\alpha)} \frac{g}{\rho^{1+\lambda}} \frac{H}{x_{F}} \frac{\partial \zeta}{\partial y}\right|_{y=0}=-\left.f \rho^{1-\mu} C \frac{H}{x_{F}} \frac{\partial \zeta}{\partial y}\right|_{y=0}
$$

Making use of eq. (19), setting $y=0$, gives

$$
\left.\frac{\partial \zeta}{\partial y}\right|_{y=0}=-(1-\varepsilon) \frac{1}{x_{F} H^{\varepsilon}} \frac{\partial}{\partial s}\left(x_{F} H^{\varepsilon}\right)
$$

to order $\varepsilon$ accuracy. Expansion through $\varepsilon^{2}$ gives an additional term $\frac{\varepsilon^{2}}{4} \frac{\partial}{\partial s}\left(\frac{1}{H} \frac{\partial H}{\partial s}\right)$ on the right hand side of eq. (27). Plugging eq. (27) (through first order) into eq. (26) gives

$$
F=f \rho^{1-\mu} C \frac{H}{x_{F}}(1-\varepsilon) \frac{1}{x_{F} H^{\varepsilon}} \frac{\partial}{\partial s}\left(x_{F} H^{\varepsilon}\right)=f \rho^{1-\mu}(1-\varepsilon) \frac{\partial}{\partial t}\left(x_{F} H^{\varepsilon}\right)
$$

for the absorbed flux. Note that this explicitly guarantees energy conservation since the total internal energy is

$$
E=\int_{0}^{x} \rho e d x=f \rho^{1-\mu} x_{F} H^{\varepsilon} \int_{0} \zeta^{\varepsilon} d y=f \rho^{1-\mu} x_{F} H^{\varepsilon}(1-\varepsilon)
$$

through first order in $\varepsilon$. Comparing eq.(28) and eq.(29) shows that $F=\frac{\partial E}{\partial t}$ as required.

Returning to eq.(20), we can obtain an explicit expression for the profile by substituting eq.(21) for the heat front position. The profile, through order $\varepsilon$ is given by

$$
\zeta=\left((1-y)\left(1+\frac{\varepsilon}{2}\left(1-\frac{1}{H} \frac{\partial H}{\partial s}\right) y\right)\right)^{\frac{1}{1-\varepsilon}}
$$


From eq. (30) we see that rising surface temperature, $\frac{\partial H}{\partial s}>0$, causes the $\zeta$ profile to have positive curvature, i.e. steeper slope near the surface than near the front, while a cooling surface has the opposite effect.

We can compare our analytic solutions to numerical results from the radiationhydrodynamics code HYDRA [6]. For this purpose we use a fit to the opacity and equation of state for gold in the temperature range $1-2 \mathrm{HeV}(1 \mathrm{HeV}=100 \mathrm{eV})$ with temperature in $\mathrm{HeV}$ units and $\rho$ in $\mathrm{g} / \mathrm{cc}$.

$$
\begin{aligned}
& f=3.4 \mathrm{MJ} / \mathrm{g} \quad \beta=1.6 \quad \mu=0.14 \\
& g=\frac{1}{7200 .} \mathrm{g} / \mathrm{cm}^{2} \quad \alpha=1.5 \quad \lambda=0.2
\end{aligned}
$$

If time is in ns units, then $\sigma=1.03 \times 10^{-2} \mathrm{MJ} / \mathrm{ns} / \mathrm{cm}^{2}$. For these parameters, $\varepsilon=0.291$ and the constant $\mathrm{C}$ is $4.08 \times 10^{-7} / \rho^{2.06} \mathrm{~cm}^{2} / \mathrm{ns}$. In Fig. 2 we compare the heat front position from eq. (25) and absorbed flux from eq. (28) with the code, for the indicated temperature drive. The surface temperature differs slightly from the incident flux temperature due to the Milne effect, as discussed below. Comparisons were made employing the actual surface temperature. Figure 3 shows the numerical and analytic spatial profiles for $\zeta$ at $\mathbf{t}$ $=1.5 \mathrm{~ns}$. Hydrodynamic motion was turned off for the simulation, which solved the grey diffusion equation employing the power law opacity and internal energy model specified in eq. (31).

\section{B. Comparison with exact analytic ('Henyey") solutions}

As a test of our solution to Eq. (7), namely Eq. (30) supplemented by Eq. (25), we compare our predictions for two special cases in which particular choices for the time 
behavior of the surface temperature $T_{S}$ lead to exact analytic solutions to Eq. (7). These solutions were found by Louis Henyey in 1954. Since they are unpublished we present their derivation in Appendix A. Here we quote the results and compare them (suitably expanded in $\varepsilon$ ) to our solution. Henyey considered the quantity $U(x, t)=T^{4+\alpha-\beta}(x, t)$ $\left(=H^{l-\varepsilon}(t) \zeta^{l-\varepsilon}(x, t)\right.$ in our notation $)$. He set out to find that choice of $T_{S}(t)$ that can lead to an exact solution to Eq. (7) for $T(x, t)$ such that $U(x, t)$ is in the form

$$
U(x, t)=a(t)+b(t) x+c(t) x^{2} .
$$

Henyey found two such choices for $T_{s}(t)$ and their associated exact solutions for $U(x, t)$.

The first special case is the simple case of $c(t)=0$, (see Appendix A) for which there is an exact solution:

$$
a(t)=T_{0}{ }^{4+\alpha-\beta} t \text { and } b(t)=-(l-\varepsilon)^{I / 2} C^{-1 / 2} T_{0}{ }^{(4+\alpha-\beta) / 2}
$$

(thus $b(t)$ is constant in time). This expression for $U(x, t)$ can be rewritten in terms of $T(x, t):$

$$
\begin{aligned}
& T_{H y}(x, t)=T_{o} t^{1 /(4+\alpha-\beta)}\left(1-x / x_{F H y}\right)^{1 /(4+\alpha-\beta)} \text { with } \\
& X_{F H y}(t)=(1-\varepsilon)^{-1 / 2} C^{1 / 2} T_{0}^{(4+\alpha-\beta) / 2} t .
\end{aligned}
$$

This $T_{H y}(x, t)$ solves Eq. (7) exactly. (The subscript Hy denotes exact Henyey solution).

We now check our theory's solution to Eq. (7), for that given input, namely $H^{l-\varepsilon}(t)=T_{0}{ }^{4+\alpha-\beta} t$. Inserting that value for $\mathrm{H}$ into Eq. (25) gives a "predicted"

$$
x_{F}(t)=((1+\varepsilon / 2) /(1-\varepsilon / 2))^{1 / 2} C^{1 / 2} T_{0}^{(4+\alpha-\beta) / 2} t
$$

which indeed matches $x_{F H y}$ through order $\varepsilon$. Moreover, inserting that same $H$ into Eq. (30) gives a "predicted"

$$
\zeta^{1-\varepsilon}=\left((1-y)\left(1-\left(O\left(\varepsilon^{2}\right)\right) y\right)=(1-y) \quad \text { through order } \varepsilon,\right.
$$


which indeed matches $\zeta_{H y}{ }^{l-\varepsilon}$. We note, at this point, that if $T_{S}(t)$ behaved as $t^{k}$ for arbitrary $k$, (and not just for the value $k=1 /(4+\alpha-\beta)$ as chosen above) then Eq. (30) gives

$$
\zeta^{1-\varepsilon}=(1-y)(1+\varepsilon / 2(1-[(2+\varepsilon) /(1-\varepsilon)][\beta k /(\beta k+\varepsilon)]) y)
$$

which means that $\zeta(y, t)$ no longer has any explicit time dependence (only implicit time dependence through $\mathrm{y}(\mathrm{t}))$ and is thus a self similar profile $\zeta=\zeta(y)$. The self-similarity is retained for $T_{s}(t) \sim t^{k}$ even when hydrodynamic effects are important as we see in Sec. III below.

The second special case is the full Henyey solution $(c(t) \neq 0$ ) (again, see Appendix A). We define $p=(1-\varepsilon) /(1+\varepsilon), q=1-p, K=2 C / \varepsilon p$, and a time variable $w=(K / A) t+1$. We consider a particular surface time behavior $U_{S H y}(t)=\left(B^{2} / 4 A\right) w^{-1}\left(w^{q}-1\right)$. The constants $A, B$ are arbitrary but can be related to the drive magnitude and duration. This behavior can describe an $\mathrm{x}$-ray drive source that is often quite relevant, in that it rises in time, peaks at time $w_{\text {peak }}=(K / A) t_{\text {peak }}+1=p^{-1 / q}$ to a value $U_{S \max }=\left(B^{2} / 4 A\right) p^{p / q} q$, and then falls slowly in time. In practice, we pick $t_{\text {peak }}$ and $U_{S \max }$ in order to match some relevant experimental x-ray drive, and the choice of $t_{\text {peak }}$ determines $A$ and the choice of $U_{S \operatorname{smax}}$ then determines $B^{2}$ (and $B$ is taken as the negative square root of $4 A U_{S \max } / p^{p / q} q$ ). Most importantly, this particular time behavior leads to an exact solution of Eq. (7) for $T(x, t)$, which is equivalent to:

$$
\begin{aligned}
& \left(U_{H y}(x, t) / U_{S H y}(t)\right)=1-\left\{[x(1-(x / B))] /\left[x_{F H y}\left(1-\left(x_{F H y} / B\right)\right)\right]\right\}, \text { with } \\
& x_{F H y}(t)=(-B / 2)\left(w^{q / 2}-1\right) .
\end{aligned}
$$

Note that with this convenient functional form of $U_{H y}(x, t)$ it is immediately obvious that $U_{H y}(x=0, t)=U_{S H y}(t)=H^{1-\varepsilon}(t)$, and $U_{H y}\left(x=x_{F h y}, t\right)=0$, both conditions fulfilling the requirements on $\zeta$ of Eq. (9). Note too that the shape of this $U$ profile is essentially the 
"right shoulder" of an inverted parabola in the upper right hand quadrant of $U$ ( $y$ axis), $x$ ( $x$ axis) space whose true peak lies in the upper left quadrant.

To verify that our equations predict this solution (through order $\varepsilon$ ) when given the $U_{S H y}(t)$ discussed above, is tedious but doable. We must expand some terms carefully to order $\varepsilon^{2}$. For example, the term $\left(w^{q}-1\right)$ that appears in $U_{S H y}(t)$, using $q=2 \varepsilon /(1+\varepsilon)$, is expanded to be $2 \varepsilon(1-\varepsilon) \ln w+2 \varepsilon^{2}(\ln w)^{2}$. The input to our solution, $H(t)=\left(U_{S H y}(t)\right)^{1 /(1-\varepsilon)}$ is then found by expanding in $\varepsilon$, and then integrated in time as a necessary ingredient in Eq. (25). The integral can be done analytically, order by order, and omitting the details, Eq. (25) finally yields:

$$
x_{F}(t)=-(B / 2) \varepsilon \ln w[1-\varepsilon+(\varepsilon / 2) \ln w] .
$$

But that is precisely the expansion of $x_{F H y}(t)=(-B / 2)\left(w^{q / 2}-1\right)$ through order $\varepsilon^{2}$, thus verifying our approach.

Checking Eq. (30) is considerably less tedious, as the $\varepsilon / 2$ coefficient therein allows us to restrict our attention to $H(t)$ to leading order, namely $\left(B^{2} / 4 A\right) 2 \varepsilon \ln w / w$. Thus, Eq. (30) then gives:

$$
\zeta^{1-\varepsilon}=(1-y)(1+[(\varepsilon / 2) \ln w] y)=(1-y)(1-x / B) .
$$

Meanwhile the exact solution, quoted above,

$$
\begin{aligned}
\left(U_{H y}(x, t) / U_{S H y}(t)\right)= & H^{I-\varepsilon} \zeta_{H y}{ }^{l-\varepsilon} / H^{I-\varepsilon}= \\
& \zeta_{H y}{ }^{1-\varepsilon}=1-\left\{[x(1-(x / B))] /\left[x_{F H y}\left(1-\left(x_{F H y} / B\right)\right)\right]\right\},
\end{aligned}
$$

can be expanded and, after dropping terms of order $(x / B)^{2}<\left(x_{F} / B\right)^{2}=O\left(\varepsilon^{2}\right)$, can be rewritten as $\zeta_{H y}^{1-\varepsilon}=(1-y)(1-x / B)$ thus showing agreement with our predicted solution. 


\section{B. Rapid time variation of the surface temperature}

We can extend the analysis in Sec. IIa to the case of rapid time variation of the surface temperature, for a special functional form of the time dependence. The special form is applicable, in particular to the case of low or zero net flux into the surface as could occur, for example, if the driving source of a hohlraum were turned off. Starting with eq. (13), we consider the case when $\varepsilon \frac{1}{H} \frac{\partial H}{\partial s} \zeta^{\varepsilon}$ is of order unity rather than $\varepsilon$. The leading order equation then becomes

$$
\begin{aligned}
& \frac{\partial}{\partial y}\left(\frac{\partial \zeta}{\partial y}+\frac{1}{x_{F}} \frac{\partial x_{F}}{\partial s} \zeta^{\varepsilon}\right)=2 z \\
& z=\frac{\varepsilon}{2} \frac{1}{H} \frac{\partial H}{\partial s}
\end{aligned}
$$

where the defined quantity $z$ is assumed of order unity. In analogy with the development above, we can integrate eq. (42) to find the lowest order equation for the heat front position $(1-\varepsilon) \frac{1}{x_{F}} \frac{\partial x_{F}}{\partial s}=1-z$, and the profile,

$$
\zeta=((1-y)(1-z y))^{\frac{1}{1-\varepsilon}} \cong(1-y)(1-z y)
$$

Note that we are restricted to $z<1$ to avoid $\zeta \rightarrow 0$ for $y$ between 0 and 1 . Also note that for $z=-1$ the slope of $\zeta$ vanishes at $y=0$ and there is no lowest order heat flux. We can employ the lowest order solution from eq. (43) in the terms of order $\varepsilon$ in eq. (13), and integrate to find the corrections. We make extensive use of the approximation $N^{\varepsilon}=e^{\varepsilon \ln (N)} \cong 1+\varepsilon \ln (N)$ to first order in $\varepsilon$, for any $\mathrm{N}$ of order unity. The results for the corrected heat front position and flux are 


$$
\begin{aligned}
& x_{F}{ }^{2}=\frac{(2+\varepsilon A)}{(1-\varepsilon)} C H^{-\varepsilon} \int H d t \\
& F=f \rho^{1-\mu} \frac{\partial}{\partial t}\left((1-\varepsilon B) x_{F} H^{\varepsilon}\right)
\end{aligned}
$$

The terms $A$ and $B$ appearing in eq. (44) are given by

$$
A=2\left(1+\frac{1}{z}+\frac{\left(1-z^{2}\right)}{z^{2}} \ln (1-z)\right), \quad B=2+\frac{(1-z)}{z} \ln (1-z)
$$

If $|z| \ll<1$ we recover the previous result with $A, B \rightarrow 1$. Over the range of $z$ from -1 to 1 we have

$$
0 \leq A \leq 3 \quad 2(1-\ln (2)) \leq B \leq 2
$$

The analysis we have used is only valid if $\frac{1}{z} \frac{\partial z}{\partial s}=\theta(1)$. From the definition of $z$, this demands that

$$
\frac{1}{\partial H / \partial s} \frac{\partial^{2} H}{\partial s^{2}}-\frac{1}{H} \frac{\partial H}{\partial s}=\Theta(1)
$$

but since $\frac{1}{H} \frac{\partial H}{\partial s}=\frac{2 z}{\varepsilon}=\Theta\left(\frac{1}{\varepsilon}\right)$, the two terms in eq. (47) must nearly cancel. This, in turn, requires that $z$ must be nearly constant for the analysis in this section to apply. The special time profiles are of the form

$$
\frac{1}{H} \frac{\partial H}{\partial s}=\frac{2 z}{\varepsilon}, \quad H=H_{0} e^{\frac{2 z s}{\varepsilon}}
$$

Through first order, the equation for $\mathrm{x}$ is

$$
\frac{1}{x_{F}} \frac{\partial x_{F}}{\partial s}=\frac{1+\frac{\varepsilon}{2} A}{1-\varepsilon}-z, \quad x_{F}=x_{0} e^{\left[\left(1+\frac{\varepsilon}{2} A\right) /(1-\varepsilon)-z\right] s}
$$

From the definition of the dimensionless time introduced in eq. (12) we have 


$$
\frac{d s}{d t}=\frac{C H^{1-\varepsilon}}{x_{F}^{2}}=\frac{C H_{0}^{1-\varepsilon}}{x_{0}^{2}} e^{2\left(\frac{z}{\varepsilon}-1\right) s}
$$

through first order. Eq. 50 can be integrated to find $s(t)$,

$$
s=\frac{-1}{2(z / \varepsilon-1)} \ln \left(1-2 C H_{0}^{1-\varepsilon}\left(\frac{z}{\varepsilon}-1\right) \frac{t}{x_{0}^{2}}\right)
$$

Plugging eq. (51) into eq. (48) the gives time dependent surface temperature associated with a given value of $z$.

$$
H=\frac{H_{0}}{\left(1-2 C H_{0}^{1-\varepsilon}\left(\frac{z}{\varepsilon}-1\right) \frac{t}{x_{0}^{2}}\right)^{\frac{1}{1-\frac{\varepsilon}{z}}}}
$$

Our solutions for rapid surface temperature variation only apply if the time-dependence is in accord with eq. (52).

For a cooling hohlraum or other situation where the net flux into the surface drops to low value once the driving source is extinguished, we expect that surface temperature would rapidly tend toward the form of eq. (52) with $z=-1$ after a transient phase. If we define $t_{0}=\frac{x_{0}{ }^{2}}{2 C H_{0}{ }^{1-\varepsilon}}$ as a characteristic time during which the surface was driven at surface temperature $T_{S}=H_{0}^{\frac{1}{4+\alpha}}$, with Marshak wave penetration depth $x_{0}$, then the surface cools according to

$$
H=\frac{H_{0}}{\left(1+\left(\frac{1}{\varepsilon}+1\right) \frac{t}{t_{0}}\right)^{\frac{1}{1+\varepsilon}}},
$$

i.e., the characteristic time for cooling is $t_{1}=\frac{\varepsilon t_{0}}{1+\varepsilon}$, much shorter than the initial drive duration $t_{0}$. 


\section{Arbitrary material properties}

We can also extend the analysis in Sec. Ila to the case of arbitrary temperature dependence of the internal energy and opacity, as well as arbitrary time dependence of the surface temperature. Our extended theory has the added benefit that one could include flux limits, which are usually treated through a modification of the opacity, or the radiation energy density, which is additive to the internal energy. The internal energy must not vary too fast with temperature (so that the analog of the parameter $\varepsilon$ exists) and the surface temperature variation in time must also not be so fast that the corrections discussed in Sec. IIc are important. Our starting point is eq. (1), but let us introduce a new independent variable

$$
Z=\int_{0}^{T} \frac{T^{3} d T}{K(T)}
$$

$Z(T)$ provides a single-valued mapping that can be inverted, in principal, to give $T(Z)$ once we obtain a solution for $Z(x, t)$. Note that for a power-law opacity, $K \propto T^{-\alpha}, Z \propto T^{4+\alpha} \propto \zeta$, the dependent variable used in previous sections. In terms of $Z$, eq.(1) becomes

$$
\begin{aligned}
& \frac{\partial e}{\partial t}=D \frac{\partial^{2} Z}{\partial x^{2}} \\
& D=\frac{16 \sigma}{3 \rho^{2}}
\end{aligned}
$$


We can also consider $e$ as a function of $Z$, i.e., $e(Z)=e(T(Z))$. As in earlier sections, it is helpful to transform to dependent variable $y=\frac{x}{x_{F}}$. Employing the transformation of the time derivative shown in eq. (10), we obtain

$$
x_{F}^{2} \dot{e}-x_{F} \dot{x}_{F} y \frac{\partial e}{\partial y}=D \frac{\partial^{2} Z}{\partial y^{2}}
$$

where the notation $\dot{e}=\left.\frac{\partial e}{\partial t}\right|_{y}$ has been introduced. This can also be expressed as

$$
\left(x_{F}{ }^{2} \dot{Z}-x_{F} \dot{x}_{F} y \frac{\partial Z}{\partial y}\right) \frac{d e}{d Z}=D \frac{\partial^{2} Z}{\partial y^{2}}
$$

From the similarity with the Sec. Ila analysis, we expect perturbation solutions to exist when $\frac{Z}{e} \frac{d e}{d Z}<<1$. For power law opacity and internal energy, in fact, $\frac{Z}{e} \frac{d e}{d Z}=\varepsilon$. We have the same difficulty near the heat front, $y=1$, that we found in Sec. IIa, i.e., $\frac{d e}{d Z} \rightarrow \infty$ as the temperature and $Z$ vanish. Accordingly, we use the "trick" that makes the left hand side uniformly small

$$
\left(x_{F}^{2} \dot{Z}+x_{F} \dot{x}_{F}(1-y) \frac{\partial Z}{\partial y}\right) \frac{d e}{d Z}=D \frac{\partial^{2} Z}{\partial y^{2}}+x_{F} \dot{x}_{F} \frac{\partial Z}{\partial y} \frac{d e}{d Z}=\frac{\partial}{\partial y}\left(D \frac{\partial Z}{\partial y}+x_{F} \dot{x}_{F} e\right)
$$

The lowest order solution is found by setting the right hand side to zero and integrating. The boundary conditions at the heat front are that $Z$ and $\frac{\partial Z}{\partial y}$ vanish, so we find to lowest order

$$
D \frac{\partial Z}{\partial y}+x_{F} \dot{x}_{F} e=0
$$


where we have assumed that the internal energy vanishes as $T$ and $Z$ go to zero. Since $e$ is a weak function of $Z$, it is adequate to replace $e$ by $e_{s}$, the value at the surface when integrating eq. (59). This is equivalent to replacing $\zeta^{\varepsilon}$ by 1 in the low order integrals in Sec. Ila. We then find the lowest order solution

$$
Z=Z_{S}-\frac{x_{F} \dot{x}_{F} e_{s}}{D} y
$$

where $Z_{S}(t)$ is the surface value of $Z$. Applying the boundary condition at the heat front gives

$$
\begin{aligned}
& Z=Z_{S}(1-y) \\
& x_{F} \dot{x}_{F}=\frac{D Z_{S}}{e_{s}}
\end{aligned}
$$

for the lowest order profile and heat front position. Note that the familiar steady-state profile re-emerges as the lowest order solution. From eq. (61) we have to lowest order $\dot{Z}=\frac{\dot{Z}_{s}}{Z_{S}} Z$ and $\frac{\partial Z}{\partial y}=-Z_{s}$ for use on the left hand side of eq. (58). We then find

$$
\left(x_{F}{ }^{2} \frac{\dot{Z}_{S}}{Z_{S}}-x_{F} \dot{x}_{F}\right) Z \frac{d e}{d Z}=\frac{\partial}{\partial y}\left(D \frac{\partial Z}{\partial y}+x_{F} \dot{x}_{F} e\right)
$$

becomes the equation through first order. Integrating eq. (62) and applying the boundary conditions at the heat front gives

$$
-\left(x_{F}{ }^{2} \frac{\dot{Z}_{S}}{Z_{S}}-x_{F} \dot{x}_{F}\right) \int_{y} d y Z \frac{d e}{d Z}=D \frac{\partial Z}{\partial y}+x_{F} \dot{x}_{F} e
$$

Next we make use of the linear relationship between $Z$ and $y$ in eq. (61) to replace the integral over $y$ with an integral over $\mathrm{Z}$, with $d y=-\frac{d Z}{Z_{S}}$ :

$$
\left(\frac{x_{F} \dot{x}_{F}}{Z_{S}}-x_{F}{ }^{2} \frac{\dot{Z}_{S}}{Z_{S}^{2}}\right) \int_{0}^{Z} d Z^{\prime} Z^{\prime} \frac{d e\left(Z^{\prime}\right)}{d Z^{\prime}}=D \frac{\partial Z}{\partial y}+x_{F} \dot{x}_{F} e
$$


Integrating a second time gives us

$$
D\left(Z-Z_{S}\right)+x_{F} \dot{x}_{F} \int_{0} d y e=\left(\frac{x_{F} \dot{x}_{F}}{Z_{S}}-x_{F}{ }^{2} \frac{\dot{Z}_{S}}{Z_{S}^{2}}\right) \int_{0} d y \int_{0}^{T_{0}} d Z^{\prime} Z^{\prime} \frac{d e\left(Z^{\prime}\right)}{d Z^{\prime}}
$$

We find the equation for the heat front position by demanding $Z(1, t)=0$. In the first order corrections on the right hand side of eq. (65) we can again replace the integral over $y$ with an integral over $Z$. This also works for the term on the left hand side of eq. (65) since

$$
\int_{0} d y e=\int_{0} d y e_{S}+\int_{0}^{1} d y\left(e-e_{S}\right)=e_{S}+\int_{0} d y\left(e-e_{S}\right)
$$

The term $\int_{0} d y\left(e-e_{S}\right)$ is a first order correction, where we can again convert it into an integral over $Z$, so

$$
\int_{0} d y e=e_{S}+\frac{1}{Z_{S}} \int_{0}^{Z_{0}} d Z^{\prime}\left(e\left(Z^{\prime}\right)-e_{S}\right)=\frac{1}{Z_{S}} \int_{0}^{Z_{S}} d Z^{\prime} e\left(Z^{\prime}\right)
$$

Our equation for the heat front then becomes

$$
-D Z_{S}+x_{F} \dot{x}_{F} \frac{1}{Z_{S}} \int_{0}^{Z_{\beta}} d Z^{\prime} e\left(Z^{\prime}\right)=\left(\frac{x_{F} \dot{x}_{F}}{Z_{S}{ }^{2}}-x_{F}{ }^{2} \frac{\dot{Z}_{S}}{Z_{S}{ }^{3}}\right)_{0}^{Z_{\beta}} d Z \int_{0}^{T_{0}} d Z^{\prime} Z^{\prime} \frac{d e\left(Z^{\prime}\right)}{d Z^{\prime}}
$$

Regrouping terms gives

$$
x_{F} \dot{x}_{F}\left(\frac{1}{Z_{S}} \int_{0}^{Z_{s}} d Z^{\prime} e\left(Z^{\prime}\right)-\frac{1}{Z_{S}{ }^{2}} \int_{0}^{z_{p}} d Z \int_{0}^{\bar{y}} d Z^{\prime} Z^{\prime} \frac{d e\left(Z^{\prime}\right)}{d Z^{\prime}}\right)+x_{F}{ }^{2} \frac{\dot{Z}_{S}}{Z_{S}{ }^{3}} \int_{0}^{Z_{0}} d Z \int_{0}^{Z} d Z^{\prime} Z^{\prime} \frac{d e\left(Z^{\prime}\right)}{d Z^{\prime}}=D Z_{S}
$$

If we define the quantity $L$ as

$$
L(Z)=\frac{1}{Z_{S}} \int_{0}^{Z_{\beta}} d Z^{\prime} e\left(Z^{\prime}\right)-\frac{1}{Z_{S}{ }^{2}} \int_{0}^{Z_{\beta}} d Z \int_{0}^{Z} d Z^{\prime} Z^{\prime} \frac{d e\left(Z^{\prime}\right)}{d Z^{\prime}}
$$

then it is straightforward to show that eq. (69) can be expressed as

$$
x_{F} \dot{x}_{F} L+x_{F}{ }^{2} \frac{\dot{L}}{2}=D Z_{S}
$$


and eq. (71) can be integrated in time to give

$$
\begin{aligned}
& x_{F}{ }^{2}=\frac{2 D}{L} \int_{0} Z_{S} d t \\
& L=\frac{2}{Z_{S}} \int_{0}^{Z_{\beta}} d Z^{\prime} e\left(Z^{\prime}\right)\left(1-\frac{Z^{\prime}}{Z_{S}}\right)=\frac{2}{Z_{S}} \int_{0}^{T} d T^{\prime} T^{\prime 3} \frac{e\left(T^{\prime}\right)}{K\left(T^{\prime}\right)}\left(1-\frac{1}{Z_{S}} \int_{0}^{T_{0}^{\prime}} d T^{\prime \prime} \frac{T^{\prime \prime 3}}{K\left(T^{\prime \prime}\right)}\right) \\
& Z_{S}=\int_{0}^{T_{0}} d T^{\prime} \frac{T^{\prime 3}}{K\left(T^{\prime}\right)}
\end{aligned}
$$

where the expression for $L(Z)$ was found from eq. (70) by integration by parts, and we have included the explicit transformation back to temperature. The heat front position is then known for any specified $K(T), e(T)$ and time-dependent surface temperature $T_{s}(t)$.

To calculate the heat flux

$$
F=-\left.\frac{4}{3} \frac{1}{K \rho} \frac{\partial \sigma T^{4}}{\partial x}\right|_{x=0}=-\left.\frac{D \rho}{x_{F}} \frac{\partial Z}{\partial y}\right|_{y=0}
$$

we make use of our first order expression in eq. (64)

$$
F=\rho e_{S} \dot{x}_{F}-\rho\left(\frac{\dot{x}_{F}}{Z_{S}}-x_{F} \frac{\dot{Z}_{S}}{Z_{S}^{2}}\right)_{0}^{Z_{f}} d Z^{\prime} Z^{\prime} \frac{d e\left(Z^{\prime}\right)}{d Z^{\prime}}
$$

Integrating by parts and regrouping terms we find

$$
F=\rho \frac{\partial}{\partial t}\left(x_{F} \frac{1}{Z_{S}} \int_{0}^{Z_{f}} d Z^{\prime} e\left(Z^{\prime}\right)\right)=\rho \frac{\partial}{\partial t}\left(x_{F} \frac{1}{Z_{S}} \int_{0}^{T} d T^{\prime} \frac{T^{\prime 3}}{K\left(T^{\prime}\right)} e\left(T^{\prime}\right)\right)
$$

Once again we find that energy conservation is guaranteed through first order since, by eq. (67) we have $\rho x_{F} \frac{1}{Z_{S}} \int_{0}^{Z_{5}} d Z^{\prime} e\left(Z^{\prime}\right)=\rho \int_{0}^{x} d x e=E$ and eq. (75) is equivalent to $F=\dot{E}$. 


\section{SUBSONIC WAVES}

In this section we construct perturbation solutions to the subsonic equations for the case that the surface temperature varies as $t^{k}$, where self-similar solutions exist. Our subsonic solutions include the hydrodynamic flow solution as well as values for the ablation pressure. In the subsonic case, the specific heat, (pressure/density) and opacity are each assumed to vary as density to a small power, of order $\varepsilon$.

The general theory of self-similar, sub-sonic Marshak waves was developed in Ref. [7,8]. Ref. [7] contains a thorough description of the self-similar model and the limits of validity, in particular the approximation that the density effectively goes to infinity at the ablation front. This corresponds, physically, to the fact that the ablated density is much less than the solid material for many cases of interest, e.g. an ICF hohlraum wall.

\section{A. Basic equations}

The basic equations in Lagrangean form are

$$
\begin{aligned}
& \frac{\partial V}{\partial t}=\frac{\partial u}{\partial m} \\
& \frac{\partial u}{\partial t}=-\frac{\partial P}{\partial m} \\
& \frac{\partial e}{\partial t}+P \frac{\partial V}{\partial t}=\frac{4}{3} \frac{\partial}{\partial m} \frac{1}{K} \frac{\partial \sigma T^{4}}{\partial m}
\end{aligned}
$$


where $V=\frac{1}{\rho}$ is the specific mass, $u$ is the flow velocity, $P$ is the pressure and the mass variable $m=\int \rho d x$. The effectively-infinite density at the ablation front means that we have the boundary conditions $u, V, T \rightarrow 0$ at the heat front as well as $T(0, t)=T_{S}(t)$. We again assume power-law dependence of opacity and equation of state variables as in Sec. Ila above.

$$
e=f T^{\beta} V^{\mu}, \quad \frac{1}{K}=g T^{\alpha} V^{\lambda}, \quad P=r \frac{e}{V}=r f T^{\beta} V^{\mu-1}
$$

The parameter $\mu$ and $r$ are assumed to be of order $\varepsilon$ (typical values for gold at 1-2 HeV are $\mu=0.14$ and $r=0.25$ ). We use a formulation of the equations from Munro [9] that allows a somewhat more general equation of state than employed in Ref. [7]. Employing the self-similar ansatz, the quantity $y=\frac{m}{m_{F}}$, with $m_{F}$ the mass coordinate of the heat front, becomes the similarity variable (analogous to $y=\frac{x}{x_{F}}$ in sections above). We have

$$
\begin{aligned}
& T=T_{S}\left(\frac{t}{t_{s}}\right)^{k} T(y) \quad V=V_{s}\left(\frac{t}{t_{s}}\right)^{l} V(y) \quad u=u_{S}\left(\frac{t}{t_{S}}\right)^{j} u(y) \\
& m_{F}=m_{F 0}\left(\frac{t}{t_{S}}\right)^{n}
\end{aligned}
$$

We use the same names for the dimensional and dimensionless scaled variables $T, V$, etc. to avoid a proliferation of notation. We are free to choose any convenient time scale factor $t_{S}$, (the quantitative result is, of course, independent of the choice of $t_{s}$ ). We can also specify the surface temperature $T_{S}$ and the power-law $k$, i.e. our convention is the dimensionless $T(1)=1$. The similarity exponents $n, l, j$ are found from inserting the 
similarity forms from eq. (78) into eq. (76) and demanding consistency of powers of time. The result, without expansion in $\varepsilon$ is

$$
\begin{aligned}
& n=\frac{1+\lambda-\frac{3}{2} \mu+\left(1-\frac{\mu}{2}-\varepsilon\left(1-\frac{\lambda}{2}\right)\right) q_{0}}{2+\lambda-2 \mu} \\
& l=\frac{1-(1-2 \varepsilon) q_{0}}{2+\lambda-2 \mu} \\
& j=\frac{\frac{\mu}{2}+\left(\varepsilon\left(1+\frac{\lambda}{2}\right)-\frac{\mu}{2}\right) q_{0}}{2+\lambda-2 \mu} \\
& l=1-n+j \\
& q_{0}=(4+\alpha) k
\end{aligned}
$$

We will assume $q_{0}$, the similarity exponent for $T^{4+\alpha}$, is of order unity. Inspection of eq. (79) shows that $j$ is of order $\varepsilon$ while $n$ and $l$ are of order unity with $l=1-n$ to lowest order. To avoid singular time-behavior of the density, we require $l>0$, which places limits on the allowable rate of rise of the surface temperature $\left(q_{0}<1 /(1-2 \varepsilon)\right)$. This requirement means that, to lowest order, $n \cong\left(1+q_{0}\right) / 2$ is in the range $1 / 2$ to 1 .

The equations can take on different form, depending on the choice of scale factors $u_{S}, V_{S}$ in eq. (78). If we demand that the variable $y=1$ at the heat front, we are not free to independently choose $m_{F 0}$. If we choose scale factors

$$
V_{S}=\left(\frac{r f t_{S}^{2} T_{S}^{\beta}}{m_{F 0}^{2}}\right)^{\frac{1}{2-\mu}} u_{S}=\frac{m_{F 0} V_{S}}{t_{S}}
$$

and define the dimensionless parameter $\Gamma$ by

$$
\Gamma=\frac{16}{3(4+\alpha)} \frac{g \sigma}{f m_{F 0}^{2}} T_{S}^{4+\alpha-\beta} V_{S}^{\lambda-\mu}
$$

then the equations become 


$$
\begin{aligned}
l V-n y \frac{\partial V}{\partial y} & =\frac{\partial u}{\partial y} \\
j u-n y \frac{\partial u}{\partial y} & =-\frac{\partial}{\partial y} \zeta^{\varepsilon} V^{\mu-1} \\
\left(q_{0} \varepsilon+\mu l+r l\right) \zeta^{\varepsilon} V^{\mu}-n y \frac{\partial}{\partial y} \zeta^{\varepsilon} V^{\mu}-r n y \zeta^{\varepsilon} V^{\mu-1} \frac{\partial V}{\partial y} & \\
= & \Gamma \frac{\partial}{\partial y} V^{\lambda} \frac{\partial \zeta}{\partial y}
\end{aligned}
$$

where we have introduced the dimensionless variable $\zeta=T^{4+\alpha}$, as in Sec. IIa. In contrast to earlier sections, the lowest order solution away from the front is found from

$$
V^{\lambda} \frac{\partial \zeta}{\partial y}=\text { constant }
$$

As we will see below, the lowest order solution for $V$ away from the front has $V \propto y^{-1}$, so integrating eq. (83) gives $\zeta=A+B y^{1+\lambda}$, with $A$ and $B$ determined by the boundary conditions at $y=0,1$, i.e.,

$$
\zeta=1-y^{1+\lambda}
$$

We can use eq. (84) in the first order corrections to the heat flow equation, as well as the hydro equations. Since $\zeta$ appears only as $\zeta^{\varepsilon}$ in the hydro equations, it will turn out that eq. (84) is sufficiently accurate for a hydro solution through first order. This fortunate decoupling of the hydro and heat equations allows us to use the hydro solution in the first order corrections to the heat equation.

\section{B. Hydrodynamic solution}

Starting from the hydrodynamic equations in eq. (82) and introducing the variable $W=V y$, we can write, 


$$
(1-n) \frac{W}{y}+\frac{j}{y}\left(W-\frac{u}{n}\right)=n y \frac{\partial}{\partial y} \frac{W}{y}+\frac{1}{n y} \frac{\partial}{\partial y}\left(\frac{\zeta^{\varepsilon} V^{\mu} y}{W}\right)
$$

Eq. (85) can be rearranged to give

$$
y \frac{\partial W}{\partial y}=\frac{W}{n} \frac{\left[W^{2}-\frac{\partial}{\partial y} \frac{y \zeta^{\varepsilon} V^{\mu}}{n}+j W\left(W-\frac{u}{n}\right)\right]}{\left[W^{2}-\frac{\zeta^{\varepsilon} V^{\mu}}{n^{2}}\right]}[
$$

There are two regions of singular behavior where the otherwise order $\varepsilon$ effects become large, i.e., very close to $y=0$, where $V \rightarrow \infty$ and the flow velocity $u \rightarrow \infty$ and near the heat front, $y=1$. If we are not too close to either region, eq. (86) becomes, to lowest order,

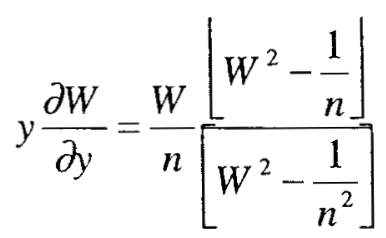

The solution of eq. (87) that is well behaved as $y$ becomes small is simply $W=\frac{1}{n^{1 / 2}}$, a constant. Note that $\mathrm{W}$ constant means that $V \propto y^{-1}$ as we assumed for the lowest order solution for $\zeta$ above. Equation (86) is somewhat awkward to deal with because of the various singularities. It is helpful to introduce the variable $H$ (different than the $H$ defined in Sec II) defined as

$$
H=\frac{1}{2}\left(W+\frac{\zeta^{\varepsilon} V^{\mu}}{n^{2} W}\right)
$$

Note that as we approach the heat front, $W \rightarrow 0, y \rightarrow 1$, we see that $H \rightarrow \frac{1}{2 n^{2}} \zeta^{\varepsilon} V^{\mu-1}=\frac{1}{2 n^{2}} P_{\text {Ablate }}$, where $P_{\text {Ablate }}$ is the dimensionless ablation pressure. The equation for $H$ can be found from eq. (85) 


$$
\frac{\partial}{\partial y} y H=\frac{(1+n)}{2 n} W+\frac{j}{2 n}\left(W-\frac{u}{n}\right)
$$

We define $\bar{H}, \bar{W}$ as the solution to eq.s (86-89) ignoring the corrections due to $j$ and $\mu$, but allowing for finite $\varepsilon$ and $\lambda$. We will find the $j$ and $\mu$ corrections later below. Eq. (89) becomes

$$
\frac{\partial}{\partial y} y \bar{H}=\frac{(1+n)}{2 n} \bar{W}=\frac{(1+n)}{2 n}\left(\bar{H}-\sqrt{\bar{H}^{2}-\frac{\zeta^{\varepsilon}}{n^{2}}}\right)
$$

where we have used

$$
\bar{W}=\bar{H}-\sqrt{\bar{H}^{2}-\frac{\zeta^{\varepsilon}}{n^{2}}}
$$

from solving eq. (88) for $\bar{W}$ in terms of $\bar{H}$. The quadratic equation (88) has two roots, but choosing the root given in eq. (91) guarantees that $\bar{W} \rightarrow 0$ at the heat front. The roots could cross if $\bar{H}^{2}=\frac{\zeta^{\varepsilon}}{n^{2}}$, but from eq. (88), that also requires $\bar{W}^{2}=\frac{\zeta^{\varepsilon}}{n^{2}}$. Inspection of eq. (86) with $\mu=j=0$ then shows that the $\bar{W}^{2}=\frac{\zeta^{\varepsilon}}{n^{2}}$ causes the denominator to vanish. As $y \rightarrow 0$ the well-behaved solution is $\bar{W} \rightarrow \frac{1}{\sqrt{n}}$, which is below the value where the denominator vanishes at $\bar{W}=\frac{1}{n}$, if $n<1 . \quad n<1$ is required to lowest order and, for the moment, we are ignoring the corrections for finite $j$, so $n$ is strictly less than 1 . In any case, we will not consider $n \geq 1$. Further inspection of eq. (86) with $\mu=j=0$ shows that, once below the curve $\bar{W}^{2}=\frac{\zeta^{\varepsilon}}{n^{2}}$, the solution remains below the curve, the denominator never vanishes and the root given in eq. (91) applies throughout the domain. 
As $y \rightarrow 0$, eq. (90) gives $\bar{H}=\frac{1+n}{2 n^{3 / 2}}$. Away from the heat front we can expand $\zeta^{\varepsilon}=1+\varepsilon \ln (\zeta)$ in eq. (91) and find the order $\varepsilon$ corrections to $\bar{H}$. We see that $\bar{H}$ is nearly constant throughout the domain with $\bar{H}=\frac{1+n}{2 n^{3 / 2}}$ plus order $\varepsilon$ corrections. The solution for $\bar{W}$ varies between $\frac{1}{\sqrt{n}}$ at $y=0$ and $\bar{H}(1)-\sqrt{\bar{H}(1)^{2}-\frac{\zeta^{\varepsilon}}{n^{2}}}=\bar{H}(1)\left(1-\sqrt{1-\frac{\zeta^{\varepsilon}}{n^{2} \bar{H}(1)^{2}}}\right) \rightarrow \frac{\zeta^{\varepsilon}}{2 n^{2} \bar{H}(1)}$ near the heat front. The asymptotic behavior suggests that

$$
\bar{W} \cong \frac{\zeta^{\varepsilon}}{\sqrt{n}}
$$

might be a reasonable approximation for $\bar{W}$ throughout the domain, even though the slope in the vicinity of the heat front is not, in general, correct. Numerical integration of eq. (90) for a wide range of $\varepsilon, n$ shows that eq. (92) is accurate to a surprising degree, apparently $\approx \mathcal{O}\left(\varepsilon^{2}\right)$. Plugging eq. (92) into eq. (90) gives us the corrections to $\bar{H}$ at the heat front

$$
\bar{H}(1)=\frac{(1+n)}{2 n} \int_{0} \bar{W} d y=\frac{(1+n)}{2 n^{3 / 2}} \int_{0} \zeta^{\varepsilon} d y \cong \frac{(1+n)}{2 n^{3 / 2}} \frac{1+\lambda}{1+\lambda+\varepsilon}
$$

where the expression for $\int_{0} \zeta^{\varepsilon} d y=\frac{1+\lambda}{1+\lambda+\varepsilon}$, using eq. (84) for $\zeta$, is accurate to about $5 \%$ for any $\lambda$, and of higher accuracy at small $\lambda$.

At this point, we can also find the lowest order solution for the flow velocity. Integrating the first equation in the set from eq. (82) we have

$$
u=\int \frac{\bar{W}}{y} d y-n \bar{W}
$$


which to lowest order, applying the boundary condition $u(1)=0$ gives simply

$$
u=\frac{\ln (y)}{\sqrt{n}}-\sqrt{n} \zeta^{\varepsilon}
$$

We are now set to include the corrections for finite $j, \mu$. Fortunately, the dominant contribution to these corrections comes from near $y=0$, and we can consider the lowest order $W$ and $H$ as constants in this region. Returning to eq. (88) and eq. (89) and assuming $W=\bar{W}+\delta W, H=\bar{H}+\delta H$ and linearizing the equation we find

$$
\begin{aligned}
& \delta W=-\frac{\delta H \bar{W}}{\bar{H}-\bar{W}}+\frac{\mu}{2 n^{2}} \frac{\ln (V) \zeta^{\varepsilon}}{\bar{H}-\bar{W}} \\
& y \frac{\partial}{\partial y} \delta H+\left[\frac{\bar{H}-\frac{(1-n)}{2 n} \bar{W}}{\bar{H}-\bar{W}}\right] \delta H=\frac{\mu}{2 n^{2}}\left(\frac{1+n}{2 n}\right) \frac{\ln (V) \zeta^{\varepsilon}}{\bar{H}-\bar{W}}+\frac{j}{2 n}\left(\bar{W}-\frac{u}{n}\right)
\end{aligned}
$$

Inserting $\bar{H}=\frac{1+n}{2 n^{3 / 2}}, \bar{W}=\frac{1}{n^{1 / 2}}$ into eq. (96) gives

$$
\begin{aligned}
& \frac{\delta W}{W}=-\frac{2 n^{3 / 2} \delta H}{1-n}+\frac{\mu}{1-n} \ln \left(1 /\left(y n^{1 / 2}\right)\right) \\
& y \frac{\partial}{\partial y} \delta H+\frac{2}{1-n} \delta H=\frac{\mu}{2 n^{3 / 2}}\left(\frac{1+n}{1-n}\right) \ln \left(1 /\left(y n^{1 / 2}\right)\right)+\frac{j}{2 n^{3 / 2}}\left(2-\frac{\ln (y)}{n}\right)
\end{aligned}
$$

where we have also made use of the lowest order flow velocity from eq. (95). Eq. (97) explicitly demonstrates the difficulties of the limit $n=1$. We can use the relation $y \frac{\partial}{\partial y} \delta H+\frac{2}{1-n} \delta H=y^{-\frac{(1+n)}{(1-n)}} \frac{\partial}{\partial y} y^{\frac{2}{1-n}} \delta H$, multiply through by $y^{\frac{(1+n)}{(1-n)}}$ and integrate to find the corrections

$$
\begin{aligned}
& \frac{\delta W}{W}=-f\left(\frac{3}{4}+\frac{1}{4 n}-\frac{\ln (y)}{2 n}\right)+\frac{\mu}{2}\left(\ln \left(1 /\left(y n^{1 / 2}\right)\right)-\frac{(1+n)}{2}\right) \\
& \frac{\delta H(1)}{H}=j \frac{(1-n)(1+3 n)}{4 n(1+n)}+\frac{\mu}{4}(1-n-\ln (n))
\end{aligned}
$$


We have evaluated $\delta H$ at the heat front since we are interested in $H(1)$ to find the ablation pressure. With eq. (98) we have the hydrodynamic solution for $H, W$ through order $\varepsilon$.

$$
\begin{aligned}
& W=\frac{\zeta^{\varepsilon}}{\sqrt{n}}\left(1+\frac{\delta W}{W}\right) \\
& H(1)=\frac{(1+n)}{2 n^{3 / 2}} \frac{1+\lambda}{1+\lambda+\varepsilon}\left(1+\frac{\delta H(1)}{H}\right)
\end{aligned}
$$

The ablation pressure is then, through order $\varepsilon$,

$$
\begin{aligned}
P_{\text {Ablate }}=2 & n^{2} H(1)= \\
& n^{1 / 2}(1+n) \frac{1+\lambda}{1+\lambda+\varepsilon}\left(1+j \frac{(1-n)(1+3 n)}{4 n(1+n)}+\frac{\mu}{4}(1-n-\ln (n))\right)
\end{aligned}
$$

Equation (99) for $W$ and the lowest order flow velocity solution in eq. (95) are of sufficient accuracy to allow us to calculate corrections to the heat flow in the next section.

\section{Heat flow solution}

Next we consider the energy transport equation from eq. (82). We start by applying a version of the "trick" discussed in Sec. II to add perfect derivative terms to both sides of the equation to remove a singularity from the left hand side,

$$
\begin{gathered}
\left(q_{0} \varepsilon+(\mu+r)(1+j)\right) \zeta^{\varepsilon} V^{\mu}+n\left(1-y^{1-\mu}\right) \frac{\partial}{\partial y} \zeta^{\varepsilon} W^{\mu}-r n y \frac{\zeta^{\varepsilon} V^{\mu}}{W} \frac{\partial W}{\partial y} \\
=\frac{\partial}{\partial y}\left(\Gamma V^{\lambda} \frac{\partial \zeta}{\partial y}+n \zeta^{\varepsilon} W^{\mu}\right)
\end{gathered}
$$

We have also used $W=V y$ where convenient in eq. (101). The term proportional to $\frac{\partial W}{\partial y}$ is problematic since it is also singular near the front. We can make use of the fact 
that $\frac{\zeta^{\varepsilon} V^{\mu}}{W}=n^{2}(2 H-W)$ from eq. (88) and use eq. (89) to manipulate the singular term into a perfect derivative term (analogous to the "trick"),

$$
\begin{gathered}
\left(q_{0} \varepsilon+(\mu+r)(1+j)\right) \zeta^{\varepsilon} V^{\mu}+n\left(1-y^{1-\mu}\right) \frac{\partial}{\partial y} \zeta^{\varepsilon} W^{\mu}+r n^{2} W^{2}\left(1+\frac{n}{2}\right)+r j n^{2} W\left(W-\frac{u}{n}\right) \\
=\frac{\partial}{\partial y}\left(\Gamma V^{\lambda} \frac{\partial \zeta}{\partial y}+n \zeta^{\varepsilon} W^{\mu}+m^{3} y\left(\frac{W^{2}}{2}+\frac{\zeta^{\varepsilon} V^{\mu}}{n^{2}}\right)\right)
\end{gathered}
$$

Except for weak, integrable singularities near $y=0$, the left hand side of eq. (102) is uniformly of order $\varepsilon$, so we can substitute lower order solutions into the left hand side and integrate to find corrected solutions. We can do this formally (and exactly) first, then plug in the lower order solutions later. Integrating eq. (102) and applying the boundary conditions $\zeta, \frac{\partial \zeta}{\partial y}, W \rightarrow 0$ at $y=1$, we have,

$$
\begin{gathered}
\left(q_{0} \varepsilon+(\mu+r)(1+j)\right) \prod_{1}^{\dagger} \zeta^{\varepsilon} V^{\mu} d y+n \int_{1}^{\infty}\left(1-y^{1-\mu}\right) \frac{\partial}{\partial y} \zeta^{\varepsilon} W^{\mu} d y \\
+r n^{2}\left(1+\frac{n}{2}\right) \int_{1}^{\dagger} W^{2} d y+r j n^{2} \int_{1}^{\dagger} W\left(W-\frac{u}{n}\right) d y \\
=\Gamma V^{\lambda} \frac{\partial \zeta}{\partial y}+n \zeta^{\varepsilon} W^{\mu}+r n^{3} y\left(\frac{W^{2}}{2}+\frac{\zeta^{\varepsilon} V^{\mu}}{n^{2}}\right)
\end{gathered}
$$

Multiplying through by $V^{-\lambda}$, integrating a second time and applying the boundary conditions $\zeta(0)=1 ., \zeta(1)=0$ gives us an equation for the quantity $\Gamma$.

$$
\begin{aligned}
& \left(q_{0} \varepsilon+(\mu+r)(1+j)\right) \int_{0} d y V^{-\lambda} \int_{1}^{t^{\prime}} d y^{\prime} \zeta^{\varepsilon} V^{\mu} \\
& +n \int_{0} d y V^{-\lambda} \int_{1} d y^{\prime}\left(1-y^{1-\mu}\right) \frac{\partial}{\partial y} \zeta^{\varepsilon} W^{\mu} \\
& \quad+r n^{2}\left(1+\frac{n}{2}\right) \int_{0} d y V^{-\lambda} \int_{1}^{\alpha} d y^{\prime} W^{2}+r j n^{2} \int_{0} d y V^{-\lambda} \int_{1}^{t} d y^{\prime} W\left(W-\frac{u}{n}\right) \\
& \quad=-\Gamma+n \int_{0} d y V^{-\lambda} \zeta^{\varepsilon} W^{\mu}+r n^{3} \int_{0} d y V^{-\lambda} y\left(\frac{W^{2}}{2}+\frac{\zeta^{\varepsilon} V^{\mu}}{n^{2}}\right)
\end{aligned}
$$


Eq. (104) can be evaluated to the desired order by substituting in the solutions for $\zeta, W$, and $V=W / y$. For instance, to lowest order, we find simply $\Gamma=n$. We obtain first order answers by substituting $\zeta=1-y^{1+\lambda}, W=\frac{1}{\sqrt{n}}$. The higher order hydro solution above allows us to evaluate many of the integrals to $\varepsilon^{2}$, which we include since it gives improved comparison with calculations, although formally the result is accurate only to first order. A complete second order solution is beyond the scope of this work. Substituting in we find,

$$
\begin{aligned}
n^{\frac{\mu-\lambda}{2}} \Gamma=\frac{\left(q_{0}\right.}{\varepsilon+(\mu+r)(1+j))} & -\frac{\varepsilon n(1+\mu)(1+\lambda)}{2 D_{1}}-\frac{\mu}{4}(j-\mu n) \\
+ & \frac{r n^{1+\frac{\mu}{2}}\left(1+\frac{n}{2}+j\right)}{2 D_{3}}+\frac{r j n^{1+\frac{\mu}{2}}\left(1+\frac{1}{2 n}\right)}{2 D_{4}} \\
& +\frac{n}{D_{5}}+\frac{r n^{2}}{4 D_{6}}+\frac{r n}{2 D_{7}}
\end{aligned}
$$

where

$$
\begin{aligned}
& D_{1}=1+\frac{3}{2} \varepsilon+\frac{3}{2} \lambda-\frac{1}{2} \mu \\
& D_{2}=1+\frac{3}{2} \varepsilon+3 \lambda+\mu \\
& D_{3}=1+3 \varepsilon+\frac{3}{2} \lambda+\frac{j}{2 n}(3 n+2)+\frac{\mu}{2}(\ln (n)+n) \\
& D_{4}=1 \\
& D_{5}=(1+\lambda)\left(1+\varepsilon+(\mu-\lambda)\left(\varepsilon+\frac{3 j}{4 n}(1+n)+\frac{\mu}{4}(\ln (n)+n-1)\right)\right) \\
& D_{6}=1+3 \varepsilon+\frac{1}{2} \lambda+\frac{j}{2 n}(3 n+2)+\frac{\mu}{2}(\ln (n)+n) \\
& D_{7}=1+3 \varepsilon+\frac{1}{2} \lambda-\frac{\mu}{2}
\end{aligned}
$$

We have assumed $\lambda$ of order $\varepsilon$ in evaluating the quantities in eq. (106). A complete second order solution would include modifications to $D_{2}$ at first order and $D_{s}$ at second 
order from the first order solution $\zeta_{1}$. The net heat flux into the surface is found from eq. (103), evaluated at $y=0$. Evaluating the required integrals gives

$$
\begin{aligned}
-\left.\Gamma V^{\lambda} \frac{\partial \zeta}{\partial y}\right|_{y=0}= & n^{1-\frac{\mu}{2}}+\frac{\left(q_{0} \varepsilon+(\mu+r)(1+j)\right) n^{-\frac{\mu}{2}}}{D_{8}} \\
& -\frac{\varepsilon n(1+\mu)(1+\lambda) n^{-\frac{\mu}{2}}}{D_{9}} \\
& +\frac{r n\left(1+\frac{n}{2}+j\right)}{D_{10}}+r j(1+n)
\end{aligned}
$$

with constants

$$
\begin{aligned}
& D_{8}=1+\varepsilon-\mu \\
& D_{9}=1+1.7 \lambda+\varepsilon+0.7 \mu \\
& D_{10}=1+2 \varepsilon+\frac{3 j}{2 n}(1+n)+\frac{\mu}{2}(\ln (n)+n-1)
\end{aligned}
$$

A consistent second order solution would include modifications of $D_{9}$ due to $\zeta_{1}$.

\section{Subsonic results}

Once we have these expressions for $\Gamma,-\left.\Gamma V^{\lambda} \frac{\partial \zeta}{\partial y}\right|_{y=0}$, we can find dimensional quantities by use of the scale factors defined in eq. (80) and the definition of $\Gamma$ in eq.

(81). For the ablated mass we find

$$
m_{F 0}=\left\{\frac{16}{3(4+\alpha)} \frac{g \sigma t_{S} T_{S}^{4+\alpha-\beta}}{f \Gamma}\left(r P_{S} f t_{S}^{2} T_{S}^{\beta}\right)^{\frac{\lambda-\mu}{2-\mu}}\right\}^{\frac{1-\frac{\mu}{2}}{2-2 \mu+\lambda}}
$$


With $m_{F O}$ from eq. (109), we can use eq. (80) to evaluate the specific volume scale factor $V_{S}=\left(\frac{r P_{S} f t_{S}{ }^{2} T_{S}{ }^{\beta}}{m_{F 0}^{2}}\right)^{\frac{1}{2-\mu}}$, where we have introduced a pressure scale factor for consistency of units, e.g., if we use megajoules for energy and nanoseconds for time, then $P_{S}=10^{-5}$ to give $V_{s}$ in cubic centimeters per gram. The dimensional heat flux is given by

$$
F=m_{F 0} f V_{s}^{\mu} T_{S}^{\beta}\left(-\left.\Gamma V^{\lambda} \frac{\partial \zeta}{\partial y}\right|_{y=0}\right)
$$

and the dimensional ablation pressure (in energy per unit volume) is

$$
P=\operatorname{rf} T_{S}^{\beta} V_{S}^{\mu-1} P_{\text {Ablate }}
$$

with the dimensionless factor $P_{\text {Ablate }}$ given in eq. (100).

We can compare the analytic theory to HYDRA simulations using the model parameters given in eq. (31). In addition to the eq. (31) parameters we must specify $r=\frac{P}{\rho e}$, and choose a temperature power-law in time. We find $r=0.25$ to be a good fit to gold in the $1-2 \mathrm{HeV}$ temperature range. Comparisons are shown for the ablated mass, absorbed flux and ablation pressure in Fig. 4(b-d) for $q_{0}=1, T_{s}=1 \mathrm{HeV}$, and using a time scale factor $t_{S}=1 \mathrm{~ns}$, i.e., $T_{s}(t)=t^{\frac{1}{4+\alpha}}=t^{0.182}$. In Fig. 5(a,b) we compare the profile of $\zeta(y)$ and $W(y)$ at $\mathrm{t}=3 \mathrm{~ns}$.

For our nominal values for $1-2 \mathrm{HeV}$ gold, we have $\beta=1.6, \alpha=1$. Sgiving $\varepsilon=0.291), \mu=0.14, \lambda=0.2, r=0.25$. These give self-similar time dependencies for the ablated mass, absorbed flux and ablation pressure of

$$
\begin{aligned}
& m(t)=m_{0} T_{S}(t)^{1.914} t^{0.5156} \\
& F(t)=F_{0} T_{S}(t)^{3.346} t^{-0.4115} \\
& P(t)=P_{0} T_{S}(t)^{2.630} t^{-0.4479}
\end{aligned}
$$


with $T_{s}(t)=T_{0} t^{k}=T_{0} t^{\frac{q_{0}}{5.5}}$ for $T_{0}$ in $\mathrm{HeV}$ and $t$ in ns. The quantities $m_{0}, F_{0}, P_{0}$ are shown as functions of $q_{0}$ in Fig. 6 (a-c). Some specific values are

$$
\begin{array}{ll}
q_{0}=0.0 & 1.0 \\
m_{0}=9.51 \times 10^{-4} \mathrm{~g} / \mathrm{cm}^{2} & m_{0}=7.13 \times 10^{-4} \mathrm{~g} / \mathrm{cm}^{2} \\
F_{0}=3.44 \times 10^{-3} \mathrm{MJ} / \mathrm{ns} / \mathrm{cm}^{2} & F_{0}=4.70 \times 10^{-3} \mathrm{MJ} / \mathrm{ns} / \mathrm{cm}^{2} \\
P_{0}=2.79 \mathrm{MB} & P_{0}=3.29 \mathrm{MB}
\end{array}
$$

\section{CONCLUSION}

In this work, we have developed a perturbation technique for solving the nonlinear radiation diffusion equation for a variety of cases of interest. The method has considerable power for generating explicit solutions to problems, in the supersonic limit, with arbitrary surface temperature time history and even arbitrary variation of material properties with temperature. Quantities of practical interest such as the depth of heat front penetration and absorbed heat flux can be readily found. In the subsonic limit, where hydrodynamic effects play a substantial role, the model is restricted to surface temperature variation $T \sim t^{k}$ and power-law temperature dependence of material properties. We are able to find, however, explicit expressions for ablated mass, the absorbed heat flux and ablation pressure in good agreement with numerical solutions.

\section{ACKNOWLEDGMENT}

The authors are grateful to M. Herrmann, M. Marinak and D. Munro for useful discussions. This work was carried out under the auspices of the U.S. Department of Energy by Lawrence Livermore National Laboratory under Contract W-7405-ENG-48. 


\section{Appendix A.}

In this Appendix, we present a more complete background to the exact analytic solutions referred to in Sec IIb. A section of an unpublished LLNL report ( UCRL-4428 (Dec. 16, 1954) by William Grassberger and Louis Henyey) reports on, and attributes, these solutions to Henyey. Since this report is unavailable in general, we present the derivation herein. Defining $U=T^{4+\alpha-\beta}$ we seek solutions of Eq. (7) for $T(x, t)$ such that $U(x, t)$ can be written as:

$$
U(x, t)=a(t)+b(t) x+c(t) x^{2}
$$

Inserting this ansatz into Eq. (7) and equating the 0,1 , and 2 powers of $x$ yields three equations:

$$
\begin{aligned}
& \frac{d a}{d t}=C \frac{(4+\alpha)}{\beta}\left\{2 a c+\left(\frac{\beta}{4+\alpha-\beta}\right) b^{2}\right\} \\
& \frac{d b}{d t}=C \frac{(4+\alpha)}{\beta}\left\{2 b c+\left(\frac{4 \beta}{4+\alpha-\beta}\right) b c\right\} \\
& \frac{d c}{d t}=C \frac{(4+\alpha)}{\beta}\left\{2 c^{2}+\left(\frac{4 \beta}{4+\alpha-\beta}\right) c^{2}\right\}
\end{aligned}
$$

As a "tune up" to solving this full set, consider the simple case of setting $c(t)=0$. Then Eq. (A.3) yields $b=$ constant, and then Eq. (A.2) yields $a(t)=C\left[(1 /(1-\varepsilon)] b^{2} t\right.$. But $a(t)$ is identical, by ansatz, to $U(x=0, t)=T_{s}^{4+\alpha-\beta}(t)$. Therefore we can write immediately 
that $T_{s}=T_{0} t^{1 / 4+\alpha \cdot \beta}$, and express $a$ and $b$ in terms of $T_{0}$, e.g., $b=C^{1 / 2}(1-\varepsilon)^{1 / 2} T_{0}^{(4+a+b / 2}$. We can then rewrite $T(x, t)$ in the useful form:

$$
\begin{aligned}
T_{H y}(x, t) & =T_{0} t^{1 /(4+\alpha-\beta)}\left(1-x / x_{F H y}\right)^{1 /(4+\alpha-\beta)} \text { with } \\
x_{F H y}(t) & =(1-\varepsilon)^{-1 / 2} C^{1 / 2} T_{0}^{(4+\alpha-\beta) / 2} t .
\end{aligned}
$$

This $T_{H y}(x, t)$ solves Eq. (7) exactly. (The subscript Hy denotes exact Henyey solution). The lesson from this simple exercise is that it takes a specific time behavior of $T_{S}(t)$ (or $\left.U_{S}(t)\right)$ to lead to an exact solution to Eq. (7) for $T(x, t)$ that would allow $U(x, t)$ to be in the form of Eq. (A.1).

To solve the full set of Eqs. (A.2)-(A.4) we start with Eq. (A.4). To simplify notation we define $p=(1-\varepsilon) /(1+\varepsilon)$, and $K=2 C / \varepsilon p$. Then Eq (A.4) yields $d c / d t=K c^{2}$ or:

$$
c(t)=-1 /(K t+A) \quad \text { where A will be determined later. }
$$

Let us denote the quantity $(K t+A)$ as a new time variable $\tau$, so $c=-1 / \tau$. Turning now to Eq. (A.3) we see that it bears a striking resemblance to Eq. (A.4) yielding $d b / d t=K b c$ which immediately tells us that $b(t)=-B c(t)$ where $B$ is a negative constant to be determined later. Thus we can write

$$
b(t)=B / \tau
$$

With that result, Eq. (A.2) now simplifies slightly:

$$
\frac{d a}{d t}=C \frac{(4+\alpha)}{\beta}\left\{2 a c+\left(\frac{\beta}{4+\alpha-\beta}\right) B^{2} c^{2}\right\}
$$

Equation (A.9) has a particular solution: $a(t)=B^{2} c(t) / 4$ (which makes it look like Eq. (A.4) again) as well as a homogeneous solution $a(t)=\gamma \tau^{p}$ where $\gamma$ will be determined later. Thus, in sum;

$$
a(t)=\left(-B^{2} \tau^{l} / 4\right)+\gamma \tau^{-p}, \quad \text { and therefore; }
$$




$$
U_{H y}(x, t)=\left[\left(-B^{2} \tau^{-1} / 4\right)+\gamma \tau^{-p}\right]+\left(B \tau^{-1}\right) x-\left(\tau^{-1}\right) x^{2}
$$

If we insist that $U(0, t)$ behaves in a reasonable way, namely that $U(0, t=0)=0$ then $\gamma$ is determined and must be:

$$
\gamma=B^{2} A^{-q} / 4 \quad \text { where } q=1-p
$$

Defining a new time variable $w=\tau / A=(K / A) t+I$ we can now write

$$
U_{S H y}(x=0, w)=\left(B^{2} / 4 A\right) w^{-1}\left(w^{q}-1\right)
$$

This is a surface source that rises from zero, and at a time $t_{\text {peak. }}$ which is given by $w_{\text {peak }}=$ $(K / A) t_{\text {peak }}+1=p^{-(1 / q)}$, reaches a maximum value, $U_{\max }=\left(B^{2} / 4 A\right) p^{(p / q)} q$, and then falls off at later times. It is this particular surface source, $U_{S H y}(x=0, t)$, that leads to the exact solution given by Eq. (A.11). To apply this formula to situations of experimental interest the user has the freedom to pick $A$ in order to match the experimental drive's $t_{\text {peak }}$, and then to pick $B$ to match the experiment's peak drive. (This is still no guarantee that the exact shape of the rise and fall of the experimental drive will match Eq. (A.13) but it might be close enough in many a situation.)

The heat front velocity $x_{F}$ is defined by $U_{H y}\left(x=x_{F}, t\right)=0$. So, finding the root of the quadratic equation $U_{H y}\left(x_{F}, t\right)=0$ from (Eq. A.11) we find:

$$
x_{F H y}(t)=(-B / 2)\left(w^{q / 2}-1\right)
$$

Using this expression, we can manipulate Eq. (A.13) into the following elegant form:

$$
\frac{U_{H y}(x, t)}{U_{S H y}(0, t)}=1-\left(\frac{x\left(1-\frac{x}{B}\right)}{x_{F}\left(1-\frac{x_{F}}{B}\right)}\right)
$$


In this form it is rather transparent that at $x=0, U=U_{S H y}(0, t)$ and that at $x=x_{F}, U=0$. We also see that the profile is that of the "right shoulder" of an inverted parabola (remember, $B$ is a negative quantity) that occupies the upper right hand quadrant of the $U-x$ plane, as it descends from the parabola's peak, which occurs in the upper left hand quadrant.

\section{Figure captions.}

Fig. 1). Temperature profile for a supersonic Marshak wave at 3ns, from the simulation shown Fig. 2.

Fig. 2a.) The temperature drive used for a supersonic test problem. b.) The heat front position vs. time from the HYDRA simulation (black) and the analytic model (red). c.) The absorbed heat flux vs. time from the HYDRA simulation (black) and the analytic model (red). The mass density used in the simulations is $0.2 \mathrm{~g} / \mathrm{cc}$.

Fig. 3). Comparison of $\zeta$ profiles from the HYDRA simulation (black) and the analytic model (red) at a.) $\mathrm{t}=1.5 \mathrm{~ns}$ and b.) $2.0 \mathrm{~ns}$.

Fig. 4). Comparison of subsonic analytic theory (red) with HYDRA simulations (black) for the temperature drive shown in a), corresponding to $q_{0}=1$, i.e. $\zeta \propto t$. Time-dependent quantities shown are b) the ablated mass, c) the absorbed flux and d) the ablation pressure. 
Fig. 5) Comparison of subsonic analytic theory (red) with HYDRA simulations (black) for the temperature drive shown in $4 \mathrm{a}$ ), corresponding to $q_{0}=1$, i.e. $\zeta \propto t$. a) shows the profile of $\zeta(y)$ at $t=3.0 \mathrm{~ns}$. b) shows $W(y)$ at $t=3.0 \mathrm{~ns}$

Fig. 6. a) $m_{0}$ in $\mathrm{g} / \mathrm{cm}^{2}$, b) $P_{0}$ in $M B$ and c) $F_{0}$ in $M J / \mathrm{cm}^{2} / \mathrm{ns}$ for the nominal material properties of gold in the range $1-2 \mathrm{HeV}$. The points shown in c) are from Ref.[10]

This work was performed under the auspices of the U.S. Department of Energy by the University of California, Lawrence Livermore National Laboratory under Contract No. W-7405-Eng-48.

\section{References}

1. R.E. Marshak, Phys. Fluids, 1, 24, (1958).

2. Ya. B. Zel' dovich and Yu. P. Raizer, "Physics of Shock Waves and High Temperature Hydrodynamic Phenomena"(Academic, New York, 1966). 
3. D. Mihalis and B.W. Mihalis, "Foundations of Radiation Hydrodynamics" (Oxford University Press, New York, 1984).

4. M.D. Rosen, Phys. Plasmas, 6, 1690, (1999).

5. F. Swenson, J.H. Hammer, Bull. Am. Phys. Soc. (2002).

6. M. M. Marinak, B.A. Remington, S.V. Weber, R.E. Tipton, S.W. Haan, K.S. Budil, O.L. Landen, J.D. Kilkenny, and R. Wallace, Phys. Rev. Lett. 74,3677 (1995).

7. R. Pakula and R. Sigel, Phys. Fluids, 28, 232, (1985).

8. N. Kaiser, J. Meyer-ter-Vehn and R. Sigel, Phys. Fluids B, 1, 1747, (1989).

9. D. Munro, private communication

10. M.D. Rosen, "Scaling law for radiation temperature," Laser Program Annual Report (1979), Lawrence Livermore National Laboratory, Livermore, CA, UCRL-50055-79, pp. 2-37 to 2-46 (unpublished). This work was reviewed in J. Lindl, Phys. Plasmas, 2, 3933, (1995). 


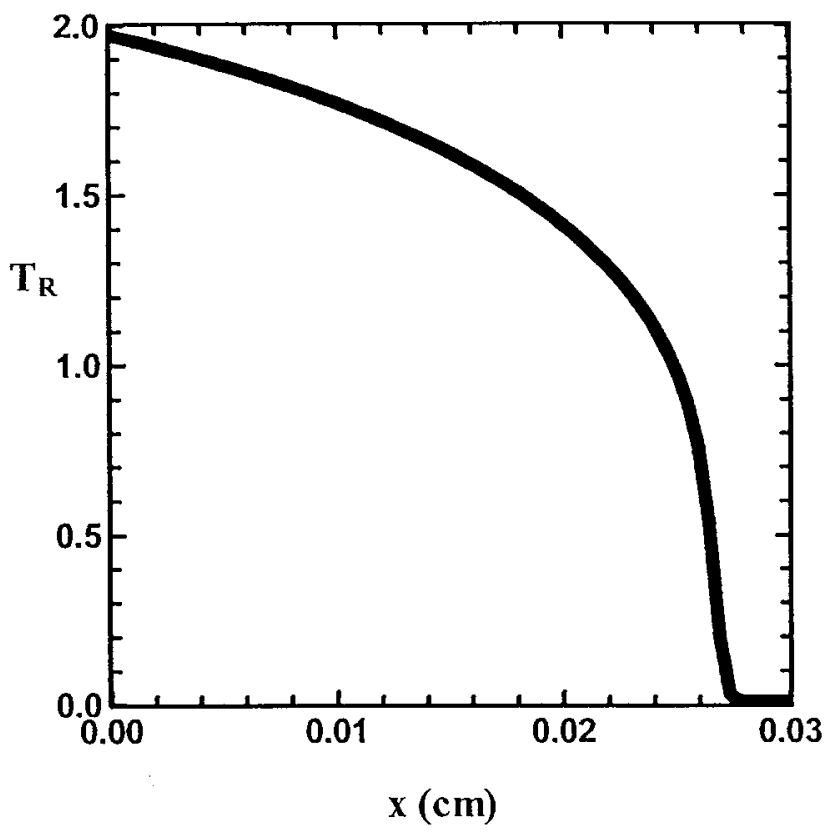

Figure 1. Hammer \& Rosen 
a)

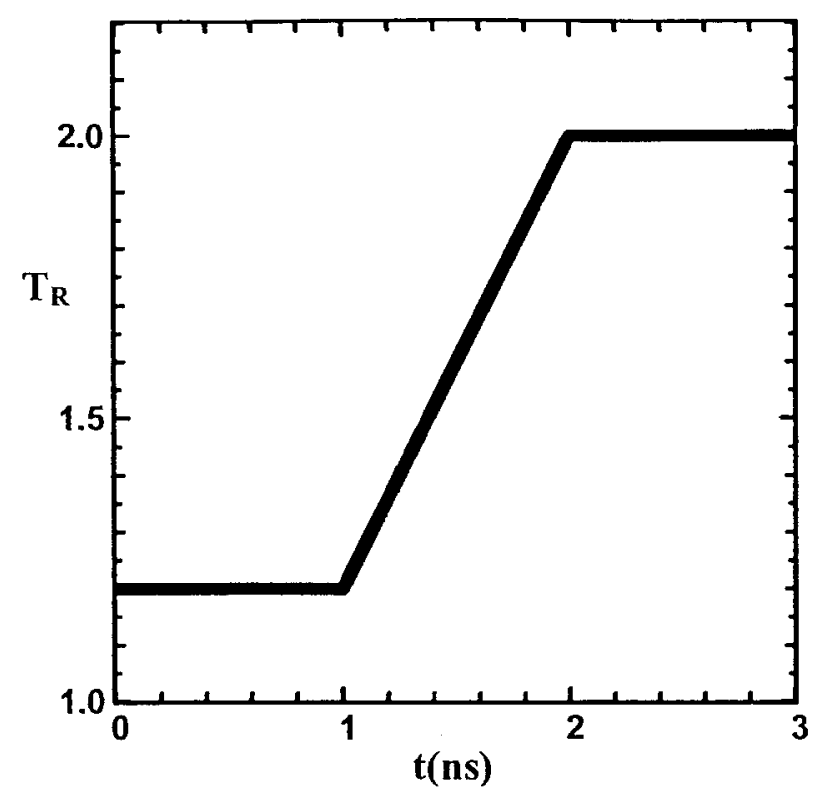

b)

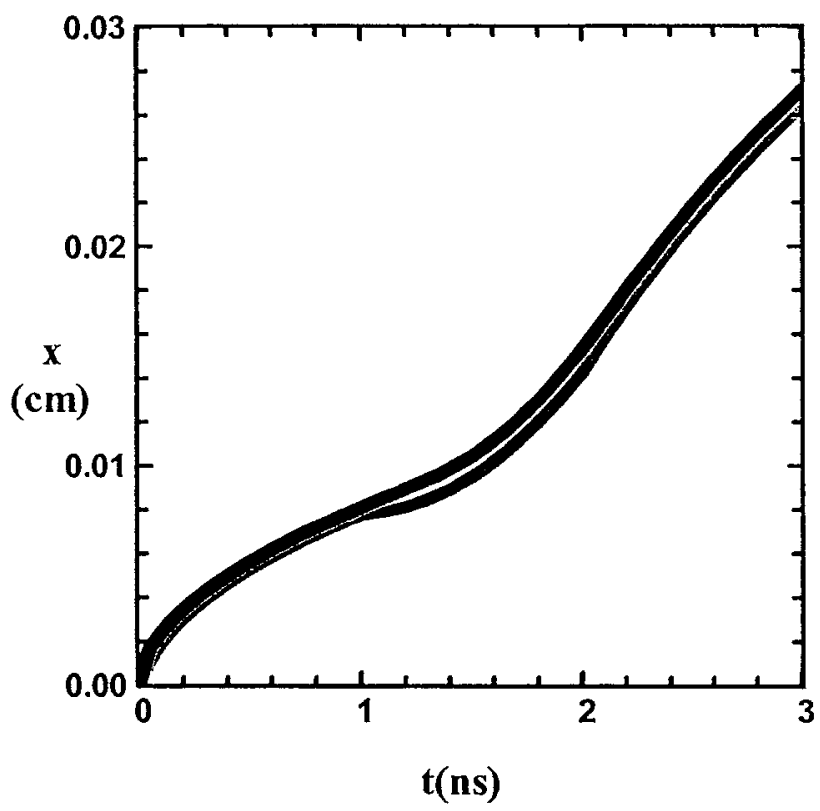

Figure 2a,2b Hammer \& Rosen 
c)

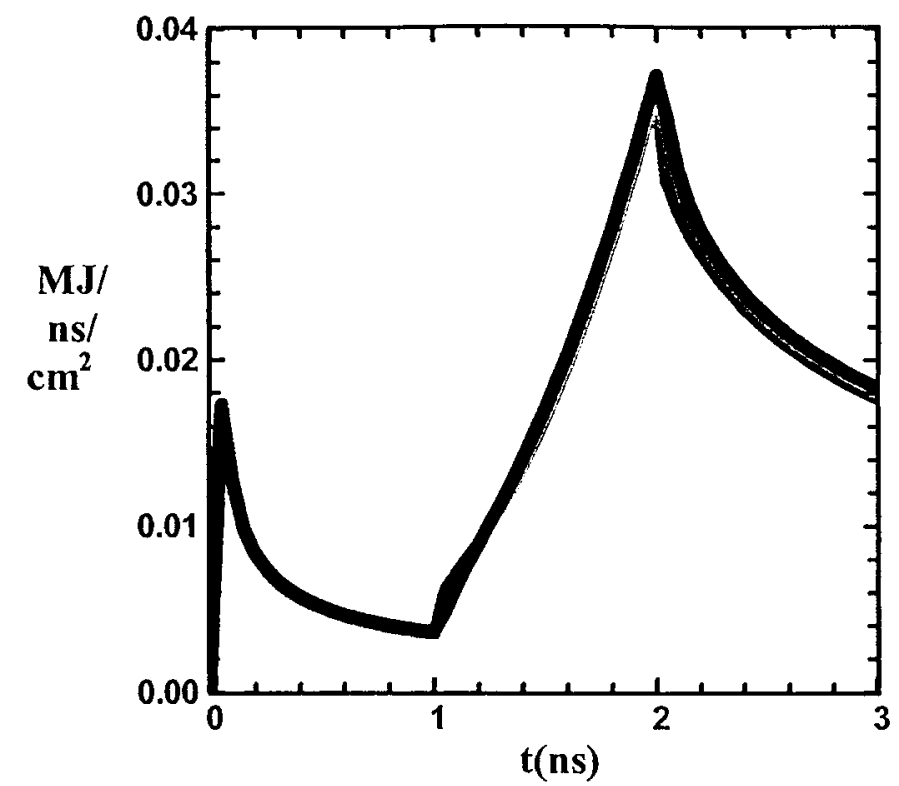

Figure 2c Hammer \& Rosen 
a)
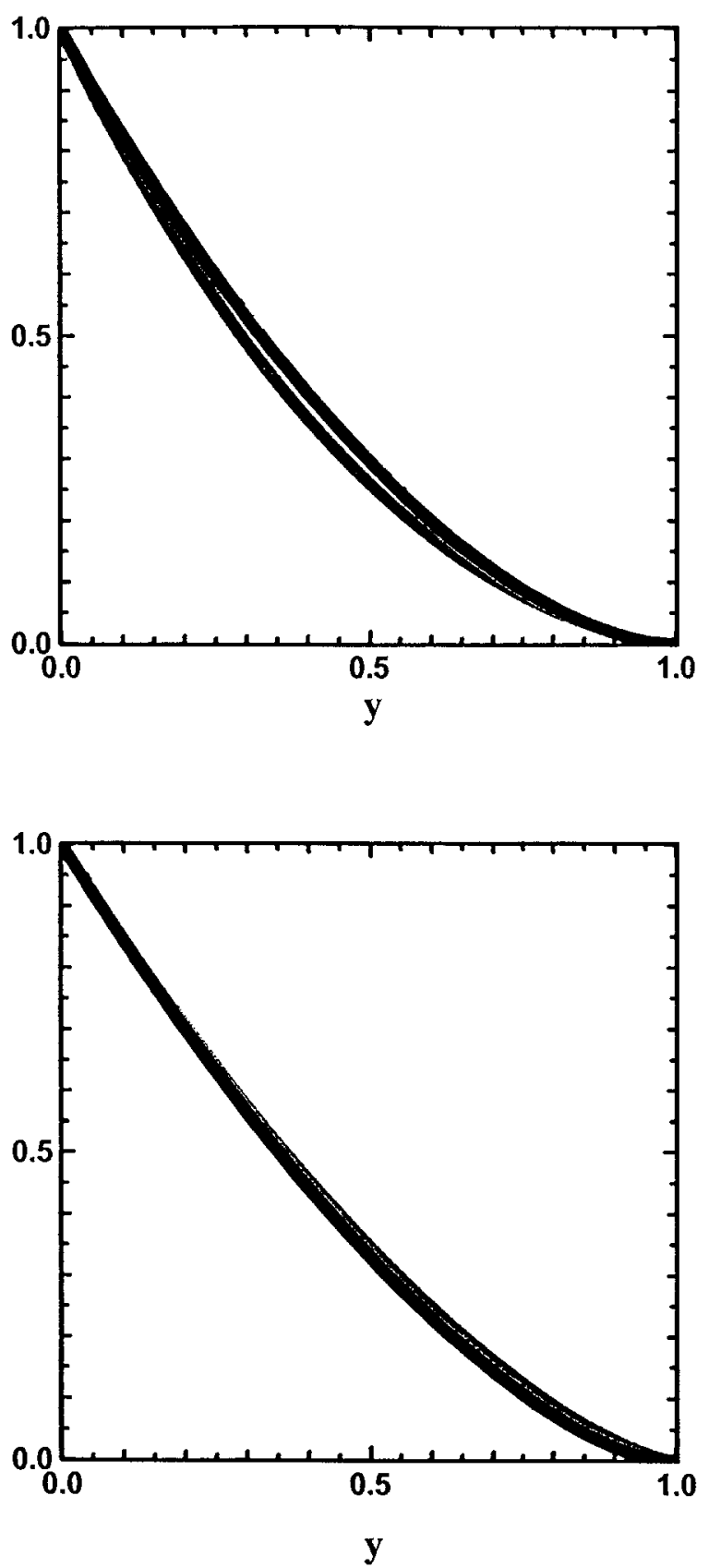

Figure 3a,b Hammer \& Rosen 
a)

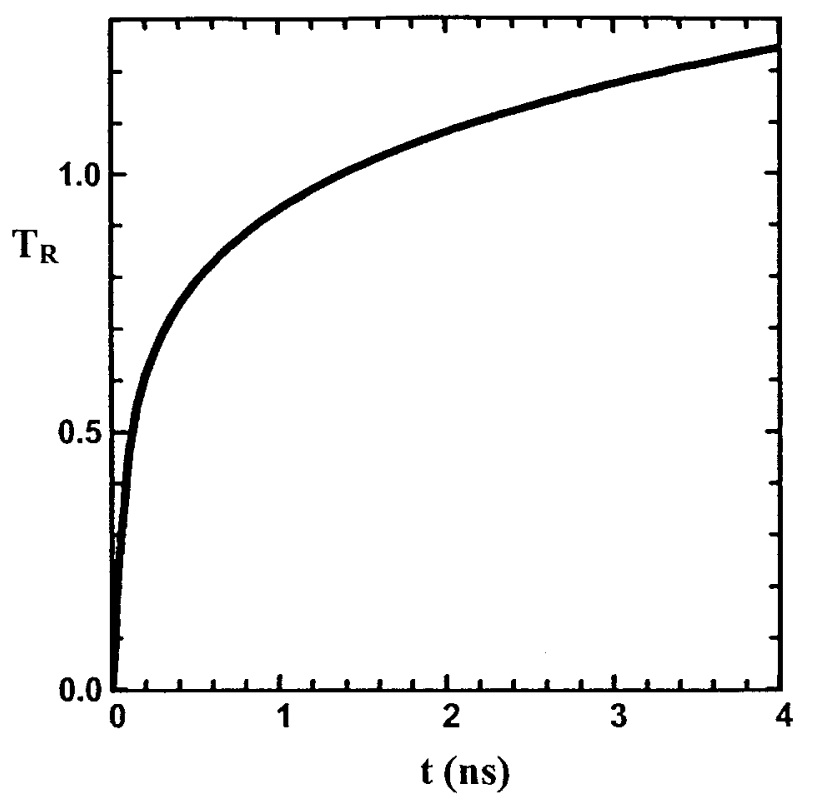

b)

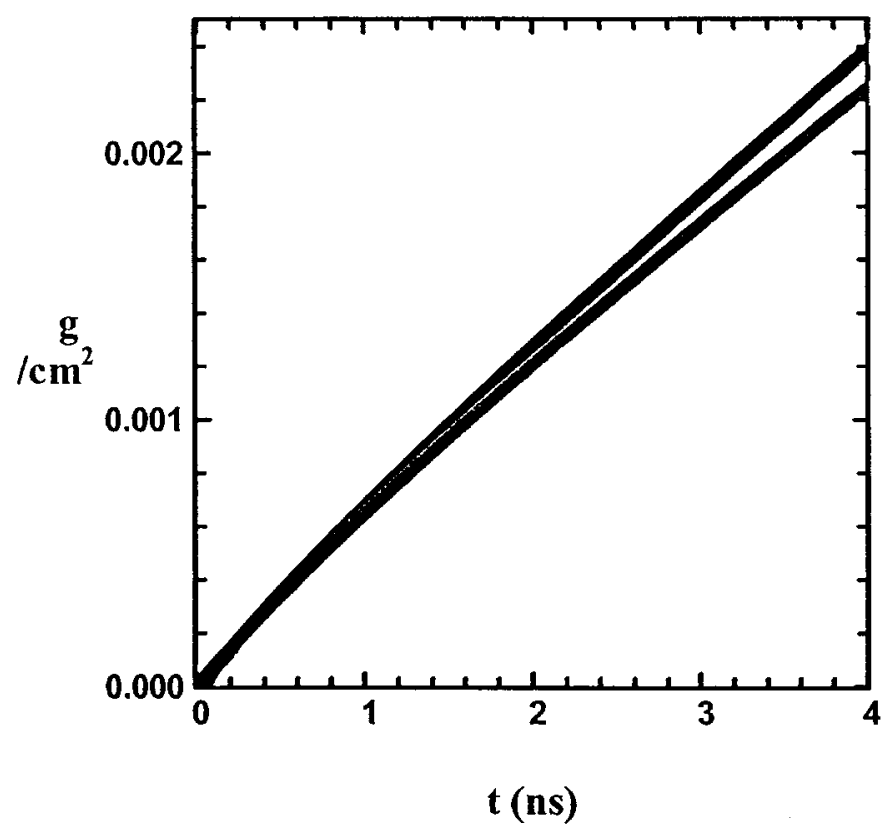

Figure 4a,b Hammer \& Rosen 
c)

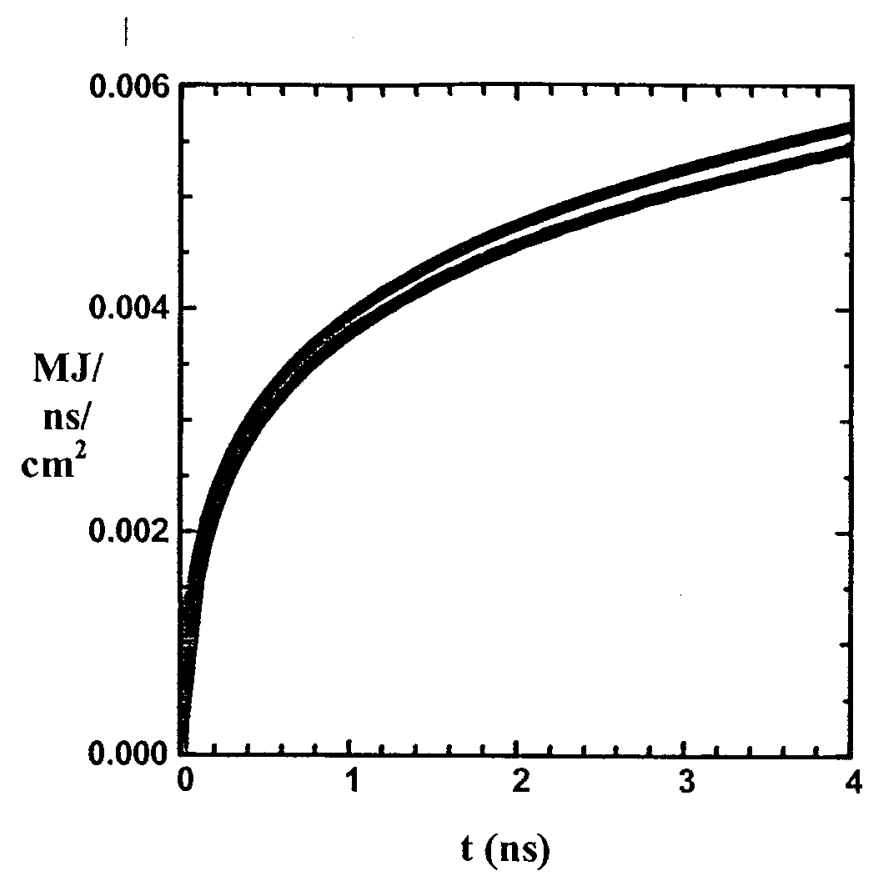

d)

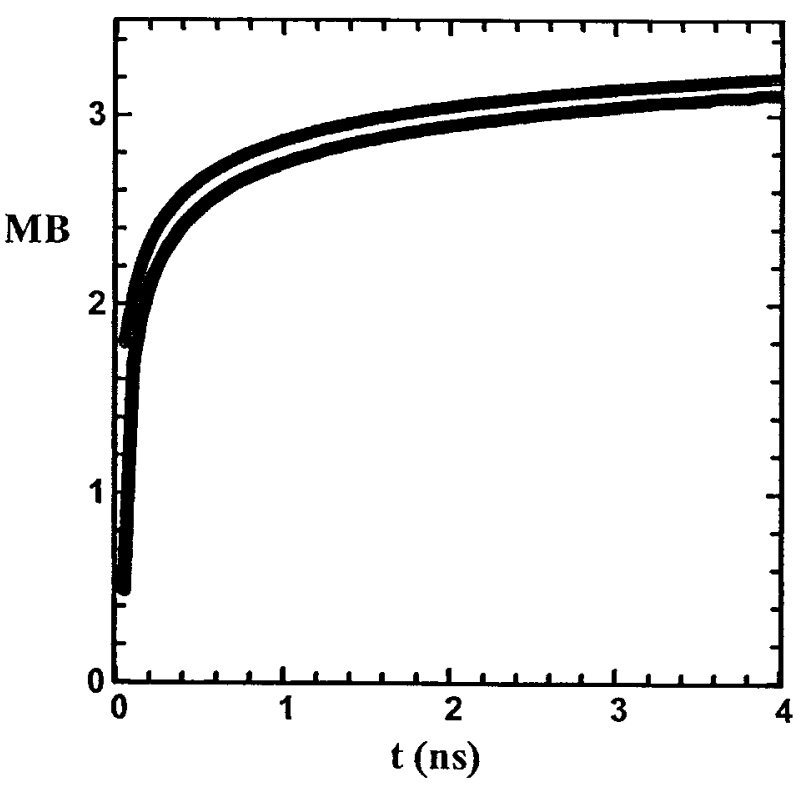

Figure 4c,d Hammer \& Rosen 
a)
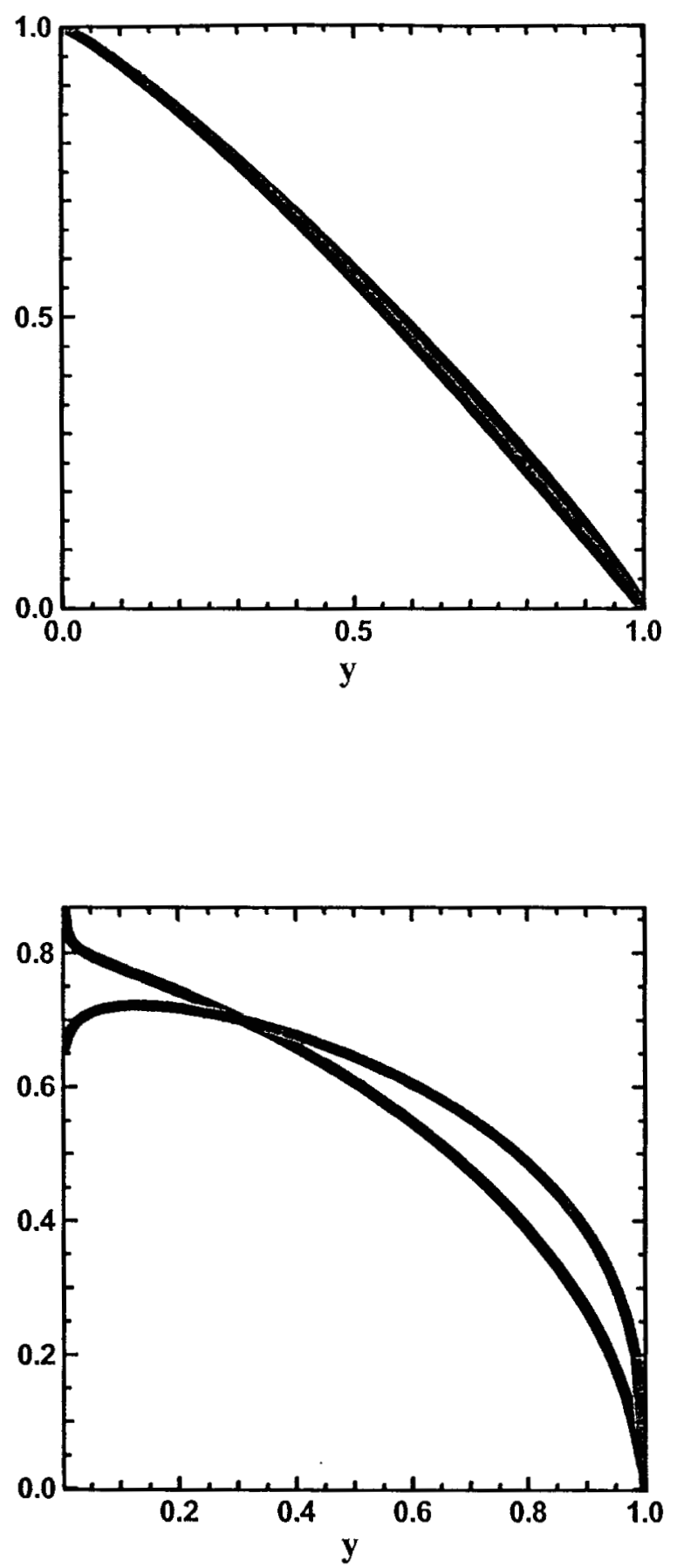

Figure 5a,b Hammer \& Rosen 
a)

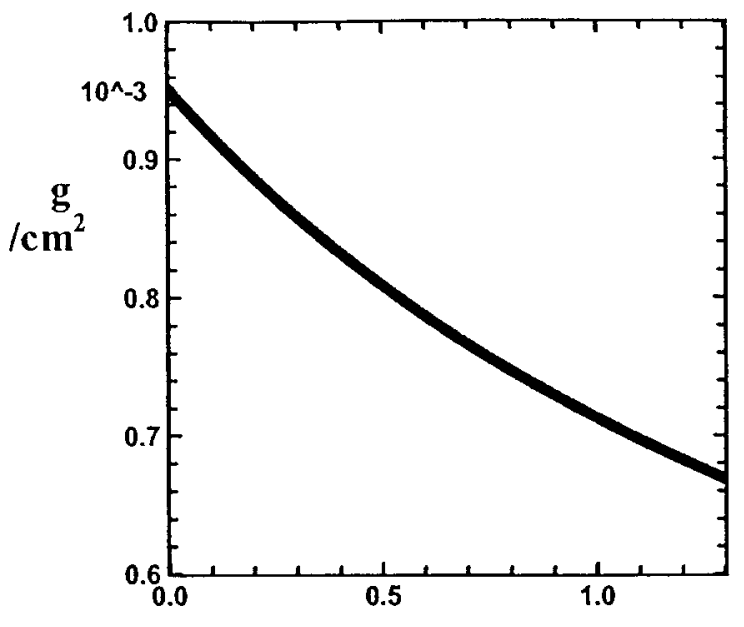

$q_{0}$

b)

c)
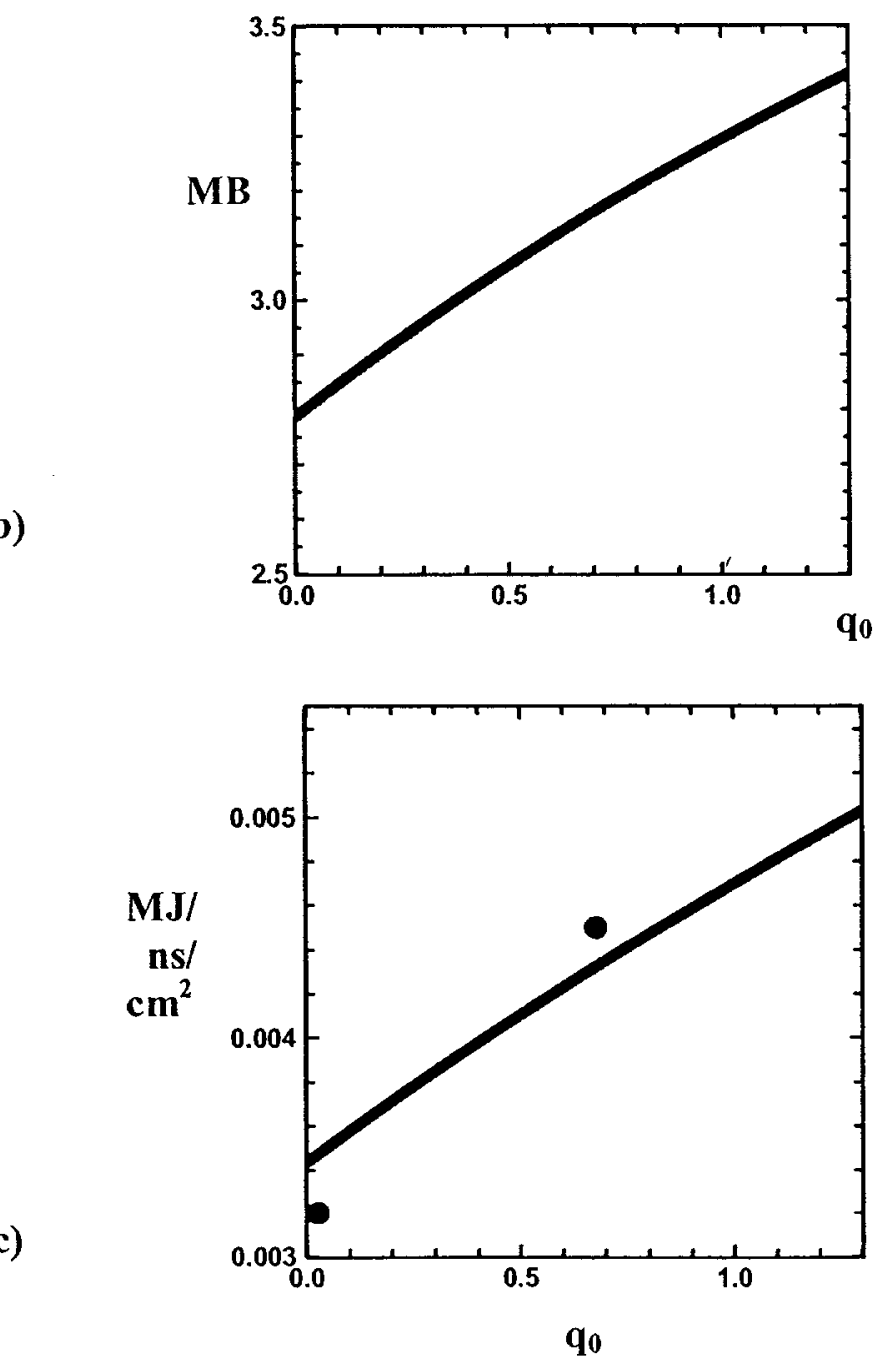

Figure 6a,bc

Hammer\&Rosen 